\title{
ENUMERATIVE GEOMETRY OF RATIONAL SPACE CURVES
}

\author{
DANIEL F. CORAY
}

[Received 5 November 1981]

1. Introduction

\section{Contents}

A. Rational space curves through a given set of points 263

$\begin{array}{ll}\text { B. Curves on quadrics } & 265\end{array}$

2. The two modes of degeneration

$\begin{array}{ll}\text { A. Incidence correspondences } & 267\end{array}$

$\begin{array}{ll}\text { B. Preliminary study of the degenerations } & 270\end{array}$

3. Multiplicities

$\begin{array}{ll}\text { A. Transversality of the fibres } & 273\end{array}$

$\begin{array}{ll}\text { B. A conjecture } & 278\end{array}$

4. Determination of $\Delta_{2,3}$

A. The Jacobian curve of the associated net 282

$\begin{array}{ll}\text { B. The curve of moduli } & 283\end{array}$

$\begin{array}{ll}\text { References } & 287\end{array}$

\section{Introduction}

\section{A. Rational space curves through a given set of points}

It is a classical result of enumerative geometry that there is precisely one twisted cubic through a set of six points in general position in $\mathbb{P}^{3}$. Several proofs are available in the literature; see, for example [15, $\$ 11$, Exercise 4; or 12, p. 186, Example 11]. The present paper is devoted to the generalization of this result to rational curves of higher degrees.

Let $m$ be a positive integer; we denote by $\mathscr{C}_{m}$ the Chow variety of all curves with degree $m$ in $\mathbb{P}_{\mathfrak{C}}^{3}$. As is well-known (cf. Lemma 2.4 ), $\mathscr{C}_{m}$ has an irreducible component $\mathscr{R}_{m}$, of dimension $4 m$, whose general element is the Chow point of a smooth rational curve. Moreover, the Chow point of any irreducible space curve with degree $m$ and geometric genus zero belongs to $\mathscr{R}_{m}$. Except for $m=1$ or 2, very little is known on the geometry of this variety. So we may also say that the object of this paper is to undertake an enumerative study of the variety $\mathscr{R}_{m}$.

We denote by $P^{\alpha} \ell^{\beta}$ the condition that a rational curve of degree $m$ should pass through $\alpha$ given points and meet $\beta$ given lines of $\mathbb{P}_{C}^{3}$, not in special position. This notation goes back to Schubert [11] and Todd [14], who investigated the case where $m=3$ of twisted cubics subject to various such constraints. It is not hard to show $(\S 2 \mathrm{~A})$ that the curves which pass through a given point $P \in \mathbb{P}^{3}$ describe a subvariety of codimension 2 in $\mathscr{R}_{m}$. Similarly, the curves which meet a given line $\ell \subset \mathbb{P}^{3}$ are represented in $\mathscr{R}_{m}$ by a subvariety of codimension 1 . Therefore, if $2 \alpha+\beta=4 m$ and general position is assumed, it is natural to expect that there is only a finite number of distinct curves, represented by points on $\mathscr{R}_{m}$, that satisfy the condition $P^{\alpha} \ell^{\beta}$. This is indeed the case (Corollary 2.4.1); we shall also denote this number by $P^{\alpha} \ell^{\beta}$. 
Furthermore, this number is not equal to zero (Lemma 2.7 and Corollary 3.2), unless $m=2, \alpha=4$, and $\beta=0$ : there is no connected curve of degree 2 through four points in general position in $\mathbb{P}^{3}$ !

These assertions are proved by considering various degenerations of the condition $P^{\alpha} \ell^{\beta}$, in which the $\alpha$ points are no longer generically chosen in $\mathbb{P}^{3}$, but are supposed to lie on some fixed smooth quadric $Q \subset \mathbb{P}^{3}$. Hence what we prove in fact is the stronger assertion that the numbers $P^{\alpha} \ell^{\beta}$ are finite even if the $\alpha$ points are not generic in $\mathbb{P}^{3}$, but only generic on $Q$. In $\S 3 \mathrm{~A}$, we shall prove even more: not only do the numbers $P^{\alpha} \ell^{\beta}$ remain finite under this specialization, but the multiplicities of the solutions are unchanged. So in particular (Corollary 3.7) the number of distinct rational curves of degree $m$ that pass through $2 m$ points is the same whether the points are generic in $\mathbb{P}^{3}$ or only generic on $Q$.

As was said at the beginning, we are primarily interested in the condition $P^{2 m}$. But it is necessary to study all the other conditions in order to settle the problem of multiplicities we have just been mentioning. Section $3 \mathrm{~B}$ is devoted to the actual determination of the numbers $P^{2 m}$. We can briefly describe how this is done: instead of studying the condition $P^{2 m}$, we first study $P^{2 m-1} \ell^{2}$. Therefore we take $2 m-1$ points $P_{1}, \ldots, P_{2 m-1}$ and two lines $L_{1}, L_{2}$ in general position in $\mathbb{P}^{3}$. If we specialize these two lines in such a way that their specializations $L_{1}^{\prime}, L_{2}^{\prime}$ meet in one point $P_{2 m}$, we see that $P^{2 m-1} \ell^{2}$ is the sum of $P^{2 m}$ and the number $V$ of curves passing through $P_{1}, \ldots, P_{2 m-1}$ and meeting $L_{1}^{\prime}$ and $L_{2}^{\prime}$ without passing through their intersection $P_{2 m}$. It will therefore suffice to determine $P^{2 m-1} \ell^{2}$ and this number $V$. As already noted above, we can assume that the $2 m$ points lie on a smooth quadric $Q$. So, in order to determine $P^{2 m-1} \ell^{2}$ and $V$, we shall specialize $L_{1}$ and $L_{2}$ into two lines $L_{1}^{\prime \prime}$ and $L_{2}^{\prime \prime}$ lying on the quadric. This will clearly introduce some multiplicities, but the number of solutions remains finite! Besides, there are two ways of choosing the specializations: either $L_{1}^{\prime \prime} \cap L_{2}^{\prime \prime}=\varnothing$ or $L_{1}^{\prime \prime} \cap L_{2}^{\prime \prime}=\left\{P_{2 m}\right\}$. Each of these possibilities corresponds to one of the two numbers to be determined. We shall see in $\$ 2 B$ that the specialized curves remain irreducible; so, by the Bézout theorem, they must lie on $Q$. Thus the numbers we are looking for can be determined by means of the geometry of quadrics!

The only difficulty with this approach is that we must determine multiplicities correctly. At the very end of the argument $(\S 3 \mathrm{~B})$, we have to introduce an assumption, which, unfortunately, is inadequately justified. If it did not hold, all the results proved before would of course still be valid, but the final formulae would look more complicated. The following theorems are therefore conditional on the validity of that assumption. (We shall designate by $\mathrm{a}^{*}$ any result whose proof depends on Conjecture $(*)$, enunciated in $\S 3 \mathrm{~B}$.

$$
\begin{array}{ll}
\text { TheOREM } 1^{*} . & P^{2 m}=(m-2)^{2}+\sum_{2 \leqslant \mu<\frac{1}{2} m}(m-2 \mu)^{2} \Delta_{\mu, m-\mu} . \\
\text { TheOREM 2*. } & P^{2 m-1} \ell^{2}=\sum_{\mu=1}^{m} \mu^{2} \Delta_{\mu, m-\mu} .
\end{array}
$$

The numbers $\Delta_{\mu, v}$ are non-negative integers, which admit of a simple geometric interpretation:

Definition. $\Delta_{\mu, v}$ is the number of irreducible curves of type $(\mu, v)$ and geometric genus zero that lie on $\mathbb{P}^{1} \times \mathbb{P}^{1}$ and pass through a generic set of $2 m-1$ points on this surface (where $m=\mu+v$ ). 
See $\$ 1 \mathrm{~B}$ for a more complete discussion. These numbers $\Delta_{\mu, \nu}$ are of a simpler nature than the $P^{\alpha} \ell^{\beta}$, because they relate to divisors on a very simple surface, as opposed to subvarieties of codimension 2. But, of course, their actual determination may raise some problems. Thus the whole of $\S 4$ is devoted to the determination of $\Delta_{2,3}$, which is the number of irreducible quintics of type $(2,3)$ that pass through nine fixed generic points of $Q$ and have two unassigned double points on this quadric. It will be found that

$$
\Delta_{2,3}=96 .
$$

Using Theorems 1 and 2, together with the elementary results of $\S 1 \mathrm{~B}$, we can thus set up a table for the first few values of the degree:

\begin{tabular}{ccc}
\hline$m$ & $P^{2 m}$ & $P^{2 m-1} \ell^{2}$ \\
\hline 1 & 1 & 1 \\
2 & 0 & 1 \\
3 & 1 & 5 \\
4 & 4 & 58 \\
5 & 105 & 1265 \\
\hline
\end{tabular}

Todd [14] gives several ways of showing that $P^{5} \ell^{2}=5$, thus confirming Theorem 2 for twisted cubics. That $P^{8}=4$ was shown by Cayley. His proof can be found in Salmon's Treatise [9, \#381], and apparently nowhere else. Our proof follows entirely different lines, since Cayley proceeds by giving an explicit construction of the four solutions. The other numbers in the table appear to be new.

It is interesting to remark that both theorems yield some lower bounds, like $P^{2 m} \geqslant(m-2)^{2}, P^{2 m-1} \ell^{2} \geqslant(m-1)^{2}$, and some congruences, like $P^{2 m} \equiv 0(\bmod 4)$ when $m$ is even. By Corollary 3.4, the other numbers $P^{\alpha} \ell^{\beta}$ are even bigger, in particular, $\ell^{4 m}$, which may be interpreted as the degree of the variety $\mathscr{R}_{m}$ in the standard embedding. (Moreover, it follows from the arguments used in $\S 3 \mathrm{~B}$ that the above inequalities would be valid even if Conjecture (*) turned out to be false!) Various upper bounds can also be obtained from the theory of Chern classes, but are not discussed in this paper.

\section{B. Curves on quadrics}

We recall briefly some properties of quadrics, which will help us to understand the significance of the numbers $\Delta_{\mu, v}$.

LEMma 1.1. Every smooth quadric surface $Q \subset \mathbb{P}^{3}$ is isomorphic to $\mathbb{P}^{1} \times \mathbb{P}^{1}$; its Picard group is therefore isomorphic to $\mathbb{Z} \oplus \mathbb{Z}$ and is generated by the classes $\mathbf{e}$ and $\mathbf{f}$ of the two families of straight lines (called 'rulings') of $Q$. The multiplicative structure obeys the following relations:

$$
\mathbf{e}^{2}=\mathbf{f}^{2}=0 ; \quad \text { e.f }=1
$$

Thus, if we fix two lines $E \in \mathbf{e}$ and $F \in \mathbf{f}$, every curve $\Gamma \subset Q$ is linearly equivalent to a (unique) divisor of the form $\mu E+\nu F$. The numbers $\mu$ and $v$ are characterized by the conditions:

$$
\begin{aligned}
\operatorname{deg} \Gamma & =\mu+v ; \\
p_{a}(\Gamma) & =(\mu-1)(v-1) .
\end{aligned}
$$


Moreover, every effective divisor of type $(\mu, v)$ is the divisor of zeros of a unique (up to scalar multiplication) bihomogeneous form $G\left(u_{0}, u_{1} ; v_{0}, v_{1}\right)$, with bidegree $(\mu, v)$. The dimension of the family of curves of type $(\mu, v)$ is consequently equal to $(\mu+1)(v+1)-1$ :

$$
\operatorname{dim}|\Gamma|=\mu \nu+\mu+v=2 \operatorname{deg} \Gamma+p_{a}(\Gamma)-1 .
$$

Proof. See, for example, [8, Chapter $1, \S 5.1$, Theorem 1; $\S 6.1$, Theorem 3 ; and Chapter 4, §2.3].

In the definition of $\Delta_{\mu, v}$ given above, we talk about a 'generic' set of points $\left\{P_{1}, \ldots, P_{2 m-1}\right\}$. In any given context, this word has a very precise meaning. For instance, no two points are on a line of $Q$, no four in a plane, no six on a twisted cubic lying on $Q$, etc. However, this notion of genericity can be properly understood only by induction on the curves of lower degree that lie on $Q$. Moreover, there will usually be some further restrictions, which are more difficult to delineate. A very efficient way to get around the difficulty is to adopt the language of Weil's Foundations: we select a field of definition $k$ for $\mathscr{R}_{m}$ and $Q$, for instance the prime field $k=\mathbb{Q}$, and assume that $\left(P_{1}, \ldots, P_{2 m-1}\right)$ is a generic point over $k$ of the symmetric product $\Sigma=Q^{\otimes 2 m-1}$. Then $\left(P_{1}, \ldots, P_{2 m-1}\right)$ does not belong to any proper subvariety of $\Sigma$ defined over $k$. Now all the restrictions that will ever occur on the choice of the points, like 'no six on a twisted cubic lying on $Q$, etc.', will correspond to some proper $k$-subvarieties of $\Sigma$. Therefore the great advantage of understanding 'generic' in the sense of Weil is that one can choose the points once and for all, and there is no need to specify the conditions each time. For simplicity, in what follows, the field over which a point is generic, or a specialization defined, will not be mentioned explicitly whenever the context is sufficiently clear.

LeMma 1.2. The numbers $\Delta_{\mu, v}$ defined in $\S 1 \mathrm{~A}$ satisfy the following relations:

$$
\begin{aligned}
& \Delta_{\mu, v}=\Delta_{v, \mu} \text { for all } \mu, v ; \\
& \Delta_{1, m-1}=1 \text { for all } m ; \\
& \Delta_{m, 0}=0 \text { for all } m>1
\end{aligned}
$$

Proof. (5) and (7) are immediate from the definition. To prove (6), we use Lemma 1.1: the curves of type $(1, m-1)$ have arithmetic genus nought, by (3), and they vary in a linear system of dimension $2 m-1$, by (4). Therefore $2 m-1$ generic points on $\mathbb{P}^{1} \times \mathbb{P}^{1}$ belong to precisely one curve of type $(1, m-1)$, which is irreducible (as follows, for example, from the Bertini theorem) and of geometric genus nought.

LEMMA 1.3. $\quad \Delta_{2,2}=12$.

Proof. By Lemma 1.1, $\Delta_{2,2}$ is the number of singular elements in a linear pencil of quartics $\Gamma$ of the first species lying on $Q$. Hence $\Delta_{2,2}$ is given by the theory of the Zeuthen-Segre invariant $[17$, Chapter $3, \S 8]$. If $S$ is a smooth surface and $\Gamma \subset S$ varies in a linear pencil of curves with only simple base points, $\uparrow$ then the number $\delta$ of singular 
elements in the pencil is given by the formula

$$
\delta=I+(\Gamma)^{2}+4 p_{a}(\Gamma) .
$$

Here $I$ denotes the Zeuthen-Segre invariant of $S$, and it is known that $I=(n-2)\left(n^{2}-2 n+2\right)$ if $S \subset \mathbb{P}^{3}$ is a smooth surface of degree $n$ (cf. [12, Chapter 9, $\S 7$, Example 3]). Hence $I=0$ in our case; moreover, $p_{a}(\Gamma)=1$ and $(\Gamma)^{2}=2 \mu v=8$; therefore $\delta=12$.

If the seven assigned base points are chosen to be generic on $Q$, all these twelve curves are irreducible, and hence $\Delta_{2,2}=\delta$. Indeed a reducible curve of type $(2,2)$ is either the union of two conics, or the union of a line and a curve of degree 3. But it follows from (4) that there is no such reducible curve through seven generic points of $Q$.

\section{The two modes of degeneration}

\section{A. Incidence correspondences}

Let $\mathscr{R}$ be any irreducible component of the Chow variety $\mathscr{C}_{m}$. We shall suppose (for simplicity) that it contains the Chow point of at least one irreducible curve. Then the curves passing through a given point $P \in \mathbb{P}^{3}$ are represented in $\mathscr{R}$ by a subvariety of codimension 2. Indeed, consider the set

$$
\mathscr{E}_{1}=\left\{(\Gamma, R) \in \mathscr{R} \times \mathbb{P}^{3} \mid R \in\langle\Gamma\rangle\right\},
$$

where $\langle\Gamma\rangle$ denotes the support of the 1 -cycle whose Chow point is $\Gamma$. This is an algebraic correspondence $[4$, Chapter $11, \S 6]$; moreover,

\section{Lemma 2.0. The incidence correspondence $\mathscr{E}_{1}$ is irreducible.}

Proof. See [18, p. 295, § I.4], which depends on the following lemma, proved in [18, p. $153, \S 21]$.

Lemma 2.0.1. Let $S$ and $S^{\prime}$ be points in some projective space. If $(\Gamma, S)$ is a specialization, over a field $k$, of $\left(\Gamma^{\prime}, S^{\prime}\right)$, then for any point $R \in\langle\Gamma\rangle$ there exists a point $R^{\prime} \in\left\langle\Gamma^{\prime}\right\rangle$ such that $(\Gamma, R, S)$ is a specialization of $\left(\Gamma^{\prime}, R^{\prime}, S^{\prime}\right)$ over $k$.

Now both projections $\varphi_{1}: \mathscr{E}_{1} \rightarrow \mathscr{R}$ and $\psi_{1}: \mathscr{E}_{1} \rightarrow \mathbb{P}^{3}$ are surjective. This is trivial for $\varphi_{1}$; therefore it suffices to show that given a generic point $P^{\prime}$ of $\mathbb{P}^{3}$ over $k$, there is a curve $\left\langle\Gamma^{\prime}\right\rangle$ through $P^{\prime}$ whose Chow point belongs to $\mathscr{R}$. Let $\Gamma$ be a generic point of $\mathscr{R}$ over $k$, and let $P$ be a generic point of $\langle\Gamma\rangle$ over $k(\Gamma)$. If $P$ is not a generic point of $\mathbb{P}^{3}$ over $k$, then $P$ is a non-generic specialization of $P^{\prime}$ over $k$. Now we can obtain a curve $\left\langle\Gamma^{\prime}\right\rangle$ through $P^{\prime}$ by performing a suitable translation on $\langle\Gamma\rangle$. It follows from the definition of Chow forms that the Chow point $\Gamma$ of $\langle\Gamma\rangle$ is a non-generic specialization of the Chow point $\Gamma^{\prime}$ of $\left\langle\Gamma^{\prime}\right\rangle$ over $k$. Therefore $\Gamma^{\prime}$ is a generic point, over $k$, of a variety $\mathscr{R}$, which contains $\mathscr{R}$ as a proper subvariety. This contradicts the fact that $\mathscr{R}$ is a component of $\mathscr{C}_{m}$.

Let $d$ be the dimension of $\mathscr{R}$; as the fibres of $\varphi_{1}$ have dimension 1 , and $\varphi_{1}$ is surjective, $\mathscr{E}_{1}$ has dimension $d+1$; and since also $\psi_{1}$ is surjective, a generic fibre of $\psi_{1}$ is of dimension $d-2$. This completes the proof that the curves passing through a 
generic $\dagger$ point of $\mathbb{P}^{3}$ are represented in $\mathscr{R}$ by a subvariety of codimension 2 , or, as we shall sometimes say, that passing through a point is a condition of weight 2 . A similar argument, using the Grassmannian $G_{1,3}$ of lines in $\mathbb{P}^{3}$ and the correspondence

$$
\mathscr{E}_{2}=\left\{(\Gamma, L) \in \mathscr{R} \times G_{1,3} \mid\langle\Gamma\rangle \cap\langle L\rangle \neq \varnothing\right\},
$$

shows that meeting a line is a condition of weight 1 . In addition,

LEMMA 2.1. The incidence correspondence $\mathscr{E}_{2}$ is irreducible.

Proof. The fibre of $\varphi_{2}: \mathscr{E}_{2} \rightarrow \mathscr{R}$ above $\Gamma \in \mathscr{R}$ is what is classically called the 'complex of secants' of $\langle\Gamma\rangle$; cf. [12, Chapter 10, $\S 2.2$, Example 10]. (A secant is any line which meets the curve; this word is therefore not synonymous with 'bisecant', or 'chord'.) Now the complex of secants of an irreducible curve $\langle\Gamma\rangle$ is irreducible, for the correspondence

$$
\mathscr{E}_{\Gamma}=\left\{(R, L) \in\langle\Gamma\rangle \times G_{1,3} \mid R \in\langle L\rangle\right\}
$$

is such that all the fibres of its first projection are irreducible, of dimension 2. Hence $\mathscr{E}_{\Gamma}$ is irreducible $[8$, Chapter $1, \S 6.3$, Theorem 8$]$, and so is the complex of secants of $\langle\Gamma\rangle$, which is nothing but the image of $\mathscr{E}_{\Gamma}$ by the second projection.

Now let $k$ be a field of definition for $\mathscr{R}$. Let $\Gamma^{\prime}$ be a generic point of $\mathscr{R}$ over $k$, and $L^{\prime}$ a generic point of the complex of secants of $\left\langle\Gamma^{\prime}\right\rangle$ over $k\left(\Gamma^{\prime}\right)$. Let $(\Gamma, L)$ be any point of $\mathscr{E}_{2}$; it is enough to show that $(\Gamma, L)$ is a specialization of $\left(\Gamma^{\prime}, L^{\prime}\right)$ over $k$. By the transitivity of specializations, this is a consequence of the following (more general) lemma.

LeMma 2.1.1. Let $T$ and $T^{\prime}$ be points in some projective space. If $(\Gamma, T)$ is a specialization, over $k$, of $\left(\Gamma^{\prime}, T^{\prime}\right)$ and if $\langle\Gamma\rangle \cap\langle L\rangle \neq \varnothing$, then there exists a line $L^{\prime} \in G_{1,3}$ such that $\left\langle\Gamma^{\prime}\right\rangle \cap\left\langle L^{\prime}\right\rangle \neq \varnothing$ and $(\Gamma, L, T)$ is a specialization of $\left(\Gamma^{\prime}, L^{\prime}, T^{\prime}\right)$ over $k$.

Proof. Let $R \in\langle\Gamma\rangle \cap\langle L\rangle$ and let $S$ be some other point of $\langle L\rangle$. Let $S^{\prime}$ be a generic point of $\mathbb{P}^{3}$ over $k\left(\Gamma^{\prime}, T^{\prime}\right)$. Then $(\Gamma, S, T)$ is a specialization of $\left(\Gamma^{\prime}, S^{\prime}, T^{\prime}\right)$ over $k[16$, Chapter 2, $\$ 1$, corollary to Theorem 5]. Moreover, by Lemma 2.0.1, we can find a point $R^{\prime} \in\left\langle\Gamma^{\prime}\right\rangle$ such that $(\Gamma, R, S, T)$ is a specialization of $\left(\Gamma^{\prime}, R^{\prime}, S^{\prime}, T^{\prime}\right)$ over $k$. Let $\left\langle L^{\prime}\right\rangle$ be the line joining $R^{\prime}$ and $S^{\prime}$. Clearly, under this specialization, $L^{\prime}$ specializes to the line $L$; hence $(\Gamma, L, T)$ is a specialization of $\left(\Gamma^{\prime}, L^{\prime}, T^{\prime}\right)$ over $k$.

Note that the points $S$ and $S^{\prime}$ in Lemma 2.0.1 do not play any role in the proof of Lemma 2.0. However, they are used in the proof of Lemma 2.1.1. Similarly, the points $T$ and $T^{\prime}$ in Lemma 2.1.1 have been introduced so as to prepare the following generalization: we define

$$
\mathscr{B}=\left(\mathbb{P}^{3}\right)^{\alpha} \times\left(G_{1,3}\right)^{\beta}
$$

(exponents are used for denoting the direct product of a variety with itself). Then the same argument as above, using a combination of Lemmas 2.0.1 and 2.1.1, shows that there is an irreducible correspondence $\mathscr{E} \subset \mathscr{R} \times \mathscr{B}$, whose general element consists of a

† The case of an arbitrary point in $\mathbb{P}^{3}$ follows again from the fact that $\mathbb{P G L}_{4}$ acts on the Chow variety. 
curve together with $\alpha$ points on it and $\beta$ lines meeting it. In the diagram

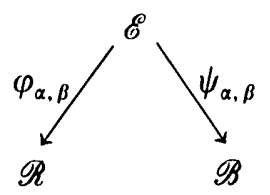

$\varphi_{\alpha, \beta}$ is clearly surjective and all its fibres are of dimension $\alpha+3 \beta$. Hence, if we assume

$$
\operatorname{dim} \mathscr{R}=2 \alpha+\beta,
$$

we have

$$
\operatorname{dim} \mathscr{E}=3 \alpha+4 \beta=\operatorname{dim} \mathscr{B} .
$$

Moreover, since $\mathscr{B}$ is normal (in fact non-singular), the principle of conservation of number applies. Hence,

Lemma 2.2. Assuming that (9) holds, we see that $\psi_{\alpha, \beta}$ is surjective if and only if one at least of its fibres is finite and non-empty. In that case the general fibre is also finite, with $\operatorname{deg} \psi_{\alpha, \beta}$ distinct points, and no finite fibre contains more than this number of points.

Proof. See [8, Chapter $2, \S 5.3$, Theorems 6 and 7]. The assumption made there, that $\psi_{\alpha, \beta}$ should be a finite morphism, is automatically satisfied above $\left\{y \in \mathscr{B} \mid \psi_{\alpha, \beta}^{-1}(y)\right.$ is finite $\}$, since proper, quasi-finite morphisms are finite, by Zariski's main theorem.

Definition. Let $\Gamma$ and $\Gamma^{\prime}$ be two effective 1 -cycles of degree $m$ in $\mathbb{P}^{3}$. We say that $\Gamma$ is a specialization of $\Gamma^{\prime}$ (over a field $k$ ) if the Chow point of $\Gamma$ on $\mathscr{C}_{m}$ is a specialization of the Chow point of $\Gamma^{\prime}$ over $k$.

In view of this definition, we now abandon the distinction between a curve $\langle\Gamma\rangle$ and its Chow point $\Gamma$. The same notation $\Gamma$ will be used for both.

Definition. Let $\Gamma=\sum \Gamma_{i}$ be an effective 1-cycle without multiple components. We define its effective genus $\pi(\Gamma)$ to be the sum of the geometric genera of all its components, $\pi(\Gamma)=\sum p_{g}\left(\Gamma_{i}\right)$.

LEMMA 2.3. Let $\Gamma$ be an effective 1-cycle without multiple components, and suppose $\Gamma$ is a specialization of an irreducible curve $\Gamma^{\prime}$. Then $\Gamma$ is connected and

$$
0 \leqslant \pi(\Gamma) \leqslant \pi\left(\Gamma^{\prime}\right) \leqslant p_{a}\left(\Gamma^{\prime}\right) \leqslant p_{a}(\Gamma) .
$$

Proof. By the so-called 'principle of degeneration' [18, p. 76, §2], $\Gamma$ is connected. The inequalities are proved in [3, Theorems 4 and 5].

In this paper we shall deal exclusively with curves of genus zero. Hence, from now on, $\mathscr{R}$ will be the variety $\mathscr{R}_{m}$ which is described in the following lemma.

Lemma 2.4. The Chow variety $\mathscr{C}_{m}$ has an irreducible component $\mathscr{R}_{m}$, of dimension $4 m$, whose general element is the Chow point of a smooth rational curve. Moreover, any irreducible space curve with degree $m$ and geometric genus zero belongs to $\mathscr{R}_{m}$. 
Proof. Any irreducible space curve with degree $m$ and geometric genus zero is the image of $\mathbb{P}^{1}$ by a map

$$
f:(t, u) \mapsto\left(a_{0} t^{m}+a_{1} t^{m-1} u+\ldots, b_{0} t^{m}+\ldots, c_{0} t^{m}+\ldots, d_{0} t^{m}+\ldots+d_{m} u^{m}\right),
$$

and in general such a map corresponds to an irreducible curve of degree $m$. (There are some exceptions, for instance if the above four polynomials have a common factor.) There are $4(m+1)$ coefficients, and hence $\infty^{4 m+3}$ maps. This defines an incidence correspondence $\mathscr{I}$, with base the open subset $U$ of $\mathbb{P}^{4 m+3}$ which parametrizes those maps $f$ for which $f\left(\mathbb{P}^{1}\right)$ is irreducible of degree $m$.

This correspondence $\mathscr{I}$ is irreducible, by the argument of Lemma 2.1. Indeed a point $P$ in a special fibre is given by its first projection $\left(a_{0}, \ldots, d_{m}\right) \in U$ and by a point $(t, u) \in \mathbb{P}^{1}$. If $\left(a_{0}^{\prime}, \ldots, d_{m}^{\prime}\right)$ is a generic point of $U$ over $k$, and $\left(t^{\prime}, u^{\prime}\right)$ is a generic point of $\mathbb{P}^{1}$ over $k\left(a_{0}^{\prime}, \ldots, d_{m}^{\prime}\right)$, then (by $[16$, Chapter $2, \S 1$, corollary to Theorem 5]) $P$ is a specialization, over $k$, of the point $P^{\prime}$ defined by $\left(a_{0}^{\prime}, \ldots, d_{m}^{\prime}\right)$ and $\left(t^{\prime}, u^{\prime}\right)$. Therefore $P^{\prime}$ is a generic point of $\mathscr{I}$ over $k$, and $\mathscr{I}$ is irreducible.

By [4, Chapter 11, $\S 6$, Theorem II, p. 115], there is a correspondence between $U$ and an irreducible subvariety $\mathscr{R}_{U}$ of the Chow variety $\mathscr{C}_{m}$, which defines the same curves as $U$. Let $\mathscr{R}_{m}$ be the closure of $\mathscr{R}_{U}$. As the $\infty^{3}$ automorphisms of $\mathbb{P}^{1}$ do not modify the image of a map $f$, the dimension of $\mathscr{R}_{m}$ is equal to $(4 m+3)-3=4 m$.

It remains for us to show that $\mathscr{R}_{m}$ is a component of $\mathscr{C}_{m}$. This follows from Lemma 2.3. Indeed, let $\Gamma$ be a smooth irreducible curve of $\mathscr{R}_{m}$, and suppose $\Gamma$ is a specialization of a curve $\Gamma^{\prime} \in \mathscr{C}_{m}$. Then $\Gamma^{\prime}$ is irreducible; and $p_{a}\left(\Gamma^{\prime}\right)=0$ by Lemma 2.3 . Therefore $\Gamma^{\prime}$ is already in $\mathscr{R}_{m}$.

COROLlary 2.4.1. The numbers $P^{\alpha} \ell^{\beta}$ are finite (but possibly equal to zero) if $2 \alpha+\beta=4 m$.

Proof. By Lemma 2.4, $\operatorname{dim} \mathscr{R}_{m}=4 m$. Hence (9) holds, and the assertion follows from Lemma 2.2 .

\section{B. Preliminary study of the degenerations}

In order to estimate the numbers $P^{2 m}$, we begin by studying the condition $P^{2 m-1} \ell^{2}$. We shall allow the constraints to degenerate in the following two ways.

Definition. (a) Let $Q \subset \mathbb{P}^{3}$ be some fixed smooth quadric surface. With the first specialization, the $2 m-1$ points $P_{1}, \ldots, P_{2 m-1}$ lie on $Q$ and the two lines $L_{1}, L_{2}$ are contained in $Q$, and both belong to the same ruling, that is, $L_{1} \cap L_{2}=\varnothing$. No further constraints are imposed.

(b) With the second specialization, the $2 m-1$ points lie on $Q$ and the two lines $L_{1}, L_{2}$ are contained in $Q$, but belong to distinct rulings, that is, $L_{1} \cap L_{2} \neq \varnothing$. No further constraints are imposed.

The fact that no further constraint is imposed will be referred to as a genericity assumption. (See the discussion in $\S 1 \mathrm{~B}$.) Specializing the constraints in this way means that we consider some special fibres of the map $\psi_{2 m-1,2}$ introduced in $\S 2 \mathrm{~A}$. Our proof of Theorem 1 depends on showing:

(i) that these fibres are finite with each of our two specializations; 
(ii) that the specialized curves are all irreducible, so that, in Case (a), they all lie on $Q$ (by the Bézout theorem); in Case (b), either they lie on $Q$, or they pass through $P_{2 m}=L_{1} \cap L_{2}$;

(iii) that we know exactly how to reckon the multiplicities of the specialized solutions, so that $P^{2 m}$ will be the difference between the weighted number of curves obtained with the first specialization (which is of course $P^{2 m-1} \ell^{2}$, thus yielding Theorem 2) and the weighted number of curves $\Gamma \subset Q$ obtained with the second specialization.

In the remainder of this section we shall restrict our attention to the first two points. Multiplicities will be discussed in $§ 3$.

Lemma 2.5. The family of all irreducible curves $\Gamma \subset Q$ with degree $m$ and geometric genus zero has dimension $2 m-1$.

Proof. The family of irreducible curves of type $(1, m-1)$ has this property (Lemma 1.1). Hence it is required to show that the dimension of any other component of our family does not exceed $2 m-1$. Now a curve $\Gamma$ in the family is the image of a map $f: \mathbb{P}^{1} \rightarrow \mathbb{P}^{1} \times \mathbb{P}^{1}$, where

$$
f(x)=\left(f_{0}(x), f_{1}(x) ; f_{0}^{\prime}(x), f_{1}^{\prime}(x)\right)
$$

consists of two pairs of polynomials, respectively of degree $\mu$ and $v$, with $\mu+v=m$, varying independently. These maps are parametrized by points of $\mathbb{P}^{2 \mu+1} \times \mathbb{P}^{2 v+1}$. Taking into account the action of the $\infty^{3}$ automorphisms of $\mathbb{P}^{1}$, we see that the dimension of any component of the family does not exceed

$$
(2 \mu+1)+(2 v+1)-3=2 m-1 .
$$

COROLlaRy 2.5.1. The numbers $\Delta_{\mu, v}$ introduced in $\S 1 \mathrm{~A}$ are finite.

Proof. Let $\left\{\mathscr{X}_{i}\right\}$ be the irreducible components of the family of all irreducible curves of type $(\mu, v)$ with geometric genus zero. Then $\operatorname{dim} \mathscr{X}_{i} \leqslant 2 m-1$. For each $i$, pick $\Gamma_{i} \in \mathscr{X}_{i}$, and let $P$ be a point of $Q$ lying off $\bigcup \Gamma_{i}$. Since passing through $P$ is a linear condition on curves of type $(\mu, v)$ and $P \notin \Gamma_{i}$, the curves passing through $P$ define a proper hyperplane section $\mathscr{Y}_{i}$ of $\mathscr{X}_{i} \subset \mathbb{P}^{\mu v+\mu+v}$, whose dimension is therefore at most $2 m-2$. Repeating this argument with the irreducible components of $\bigcup \mathscr{Y}_{i}$, we complete the proof by induction on the dimension.

Lemma 2.6. Any curve $\Gamma \in \mathscr{R}_{m}$ satisfying the first specialization of the condition $P^{2 m-1} \ell^{2}$ is irreducible and lies on $Q$. These curves form a finite, non-empty set. Hence $\psi_{2 m-1,2}$ is surjective. For $m \geqslant 2$, the same assertions hold for the curves $\Gamma \in \mathscr{R}_{m}$ which satisfy the second specialization of the condition $P^{2 m-1} \ell^{2}$ without passing through $L_{1} \cap L_{2}$.

Proof. By Lemma 2.4, $\Gamma$ is a specialization of an irreducible curve $\Gamma^{\prime}$ of genus zero. If we suppose that $\Gamma$ has no multiple component then, by Lemma 2.3,

$$
0 \leqslant \pi(\Gamma) \leqslant p_{g}\left(\Gamma^{\prime}\right)=0 .
$$

Hence every component $\Gamma_{j}$ of $\Gamma$ has geometric genus zero. This is also true if $\Gamma$ has multiple components, but another argument is needed; see [10, Lemma 5, p. 392]. 
Put $m_{j}=\operatorname{deg} \Gamma_{j}$. Since $\Gamma$ meets $Q$ in at least $2 m+1$ points, it has a component $\Gamma_{0}$ contained in $Q$, by the Bézout theorem. By Lemma 2.5 , this component can satisfy at best $P^{2 m_{0}-1} \ell^{2}$, because of the genericity assumptions made in the definition of the specializations. Similarly, any other component $\Gamma_{j} \subset Q$ can pass through at most $2 m_{j}-1$ of the points; and any component $\Gamma_{j} \not Q$ can satisfy at best $P^{2 m_{j}}$, by the Bézout theorem. These conditions do not add up to $P^{2 m-1} \ell^{2}$, unless $\Gamma_{0}$ is the only component contained in $Q$. Thus we see that $\Gamma=\Gamma_{0} \cup \Gamma_{1} \cup \ldots \cup \Gamma_{r}$, with $\Gamma_{0} \subset Q$ satisfying $P^{2 m_{0}-1} \ell^{2}$ and $\Gamma_{j} \not Q$ satisfying $P^{2 m_{j}}(j \geqslant 1)$.

Now, by Lemma 2.5 , only finitely many rational curves $\Gamma_{0}$ can be found which satisfy $P^{2 m_{0}-1} \ell^{2}$. Hence, by the genericity assumptions and the Bézout theorem again, we have $\Gamma_{0} \cap\left(\Gamma_{1} \cup \ldots \cup \Gamma_{r}\right)=\varnothing$. But, by Lemma $2.3, \Gamma$ must be connected. Therefore $\Gamma=\Gamma_{0}$, that is, $\Gamma$ is irreducible and lies on $Q$. We have also seen that these curves form a finite set, which is non-empty, because there is always a curve of type $(1, m-1)$ among the solutions (unless $m=1$ with the second specialization!). By Lemma 2.2, the assertion for the first specialization implies that $\psi_{2 m-1,2}$ is surjective.

Lemma 2.7. The set of curves $\Gamma \in \mathscr{R}_{m}$ passing through $2 m$ points in general position on $Q$ is finite. If $m \neq 2$, it is also non-empty.

Proof. We may clearly assume that $m>2$. Let $\left\{P_{1}, \ldots, P_{2 m-1}\right\}$ be a generic set of $2 m-1$ points on $Q$, and let $\Gamma_{0}$ be the curve of type $(1, m-1)$ which passes through them. Let $P_{2 m}$ be a generic point of $\Gamma_{0}$ over $k\left(P_{1}, \ldots, P_{2 m-1}\right)$. By Lemma 2.5 , we know that only finitely many irreducible curves $\Gamma \in \mathscr{R}_{m}$ lying on $Q$ pass through $P_{1}, \ldots, P_{2 m-1}$. Let $F$ be the union of these curves, other than $\Gamma_{0}$. Then, by the definition of $P_{2 m}$, we have

$$
P_{2 m} \in \Gamma_{0} \backslash F \text {. }
$$

There is at least one curve $\Gamma \in \mathscr{R}_{m}$ that passes through $P_{1}, \ldots, P_{2 m}$, namely $\Gamma_{0}$. It suffices to show that there are only finitely many others. This will also imply that the morphism $\psi_{2 m, 0}$ is surjective, since we shall have found a finite, non-empty fibre (Lemma 2.2).

Suppose there are infinitely many curves $\Gamma \in \mathscr{R}_{m}$ passing through $P_{1}, \ldots, P_{2 m}$. Then the variety $\mathscr{R}_{m}$ contains a curve $\mathscr{S}$ of such solutions $\Gamma$. The incidence correspondence $\mathscr{I} \subset \mathscr{S} \times \mathbb{P}^{3}$ projects onto a surface $S \subset \mathbb{P}^{3}$ (the 'carrier variety' of $\mathscr{S}$ ), which is the union of all the curves $\Gamma$ that belong to $\mathscr{S}$. We know that $S \supset \Gamma_{0}$. However, the intersection $Q \cap S$ is not reduced to $\Gamma_{0}$. This is because $m \neq 2$ and (if $S \neq Q$ ) the divisor $Q . S$ on $Q$ is of type $(\lambda, \lambda)$ with $\lambda=\operatorname{deg} S$ : such a divisor cannot be a multiple of $\Gamma_{0}$, which is of type $(1, m-1)$.

As $T=(Q \cap S) \backslash \Gamma_{0}$ has dimension at least 1 , it is met by a generic line $L_{1}$, over $k\left(P_{1}, \ldots, P_{2 m}\right)$, of at least one of the two rulings. Pick

$$
R \in L_{1} \cap T \text {. }
$$

Since $R \in S$, we can find a curve $\Gamma \in \mathscr{S}$ which passes through $R$. As $\Gamma$ contains $P_{1}, \ldots, P_{2 m}$ and $R$, it satisfies the first specialization of $P^{2 m-1} \ell^{2}$ (on taking $L_{2}$ to be the line through $P_{2 m}$ which belongs to the same ruling as $L_{1}$ !). Thus, by Lemma 2.6, $\Gamma$ is irreducible and lies on $Q$. Therefore, by (11), $\Gamma$ is equal to $\Gamma_{0}$, since $P_{2 m} \in \Gamma \backslash F$. But this is absurd, since it follows from (12) that $R \in \Gamma \backslash \Gamma_{0}$.

$\dagger$ Note the abuse of language: in this proof, $P$ and $\ell$ refer to points and lines lying on $Q$. 
Corollary 2.7.1. $P^{2 m} \neq 0$ provided that $m \neq 2$. Equivalently, the morphism $\psi_{2 m, 0}$ is surjective for $m \neq 2$.

Corollary 2.7.2. Any curve $\Gamma \in \mathscr{R}_{m}$ passing through $2 m$ points in general position on $Q$ is irreducible.

Proof. By Lemma 2.7, if we write $m=\sum m_{i}$, there are only finitely many irreducible curves $\Gamma_{i} \in \mathscr{R}_{m_{i}}$ through $2 m_{i}$ generic points of $Q$. It can be shown that, for $2 m$ generic points on $Q$, the unions of curves one can build in this way are necessarily disconnected. By Lemma 2.3, these reducible curves are not in $\mathscr{R}_{m}$.

Perhaps the easiest way to prove the assertion is by using the action of the orthogonal group associated with the equation of $Q \subset \mathbb{P}^{3}$. Given two finite sets of irreducible curves $\Gamma_{1} \in \mathscr{R}_{m_{1}}$ and $\Gamma_{2} \in \mathscr{R}_{m_{2}}$ not lying on $Q$, one can move the second set by an element of the orthogonal group so as to disconnect it from the first set of curves (which one keeps fixed). This shows that, for $2\left(m_{1}+m_{2}\right)$ generic points, there is no connected reducible curve among the solutions.

\section{Multiplicities}

\section{A. Transversality of the fibres}

Notation. For fixed $\rho$ and $\sigma$ satisfying

$$
\rho+\sigma=2 m-1
$$

we shall denote by $C$ the condition $P^{\rho} \ell^{2 \sigma}$ that a curve $\Gamma \in \mathscr{R}_{m}$ should pass through $\rho$ generic points and meet $2 \sigma$ generic lines of $\mathbb{P}^{3}$. We shall write $C_{0}$ for the same condition, but with the $\rho$ points lying in general position on $Q$; the $2 \sigma$ lines are still supposed to be generic in $\mathbb{P}^{3}$. Similarly, we shall consider the conditions $C P, C \ell, C \ell^{2}$ and their specializations $C_{0} P, C_{0} P_{0}, C_{0} \ell, C_{0} \ell^{2}$. For instance, $C_{0} P$ is a specialization of $P^{\rho+1} \ell^{2 \sigma}$, in which all but one of the points lie on $Q$, the remaining point and the lines being generic in $\mathbb{P}^{3}$.

Lemma 3.1. The set of curves $\Gamma \in \mathscr{R}_{m}$ satisfying the condition $C P$ is finite. If it is nonempty, then the set of curves $\Gamma \in \mathscr{R}_{m}$ that satisfy either $C \ell^{2}$ or $C_{0} \ell^{2}$ is also finite and non-empty.

Proof. The dimension of $\mathscr{R}_{m}$ is $2(\rho+1)+2 \sigma=2 \rho+(2 \sigma+2)=4 m$ (Lemma 2.4). Hence, by Lemma 2.2, the set of curves $\Gamma \in \mathscr{R}_{m}$ that satisfy either $C P$ or $C \ell^{2}$ is finite, since it can be identified with a generic fibre of the map $\psi_{\rho+1,2 \sigma}$, respectively $\psi_{\rho, 2 \sigma+2}$. Therefore it is enough to show that, if $C P \neq 0$, then $C \ell^{2} \neq 0$ and $C_{0} \ell^{2}$ is finite.

Suppose $C \ell^{2}=0$. Then the set of curves $\Gamma \in \mathscr{R}_{m}$ that satisfy $C \ell$ is finite. For if it were infinite, then the union of these curves would fill at least a surface $S \subset \mathbb{P}^{3}$. A generic line $L \subset \mathbb{P}^{3}$ would meet $S$ in some points, i.e. on some curves $\Gamma$ in the family, so that $C \ell^{2} \neq 0$, a contradiction. Now the general fibre of the map $\psi_{\rho, 2 \sigma+1}$ is either infinite or empty (cf. Lemma 2.2: (10) is replaced by $\operatorname{dim} \mathscr{E}=1+\operatorname{dim} \mathscr{B}$ ). Since we have just shown that it is finite, we must have $C \ell=0$. However, this contradicts our assumption that $C P \neq 0$, which implies that given any point $R$ of a generic line $L$, there is a curve $\Gamma$ satisfying $C$ and passing through $R$, and hence meeting $L$.

This shows that $C \ell^{2} \neq 0$ and $\psi_{\rho, 2 \sigma+2}$ is surjective. Of course this implies that $C_{0} \ell^{2} \neq 0$. We have to show that this number is also finite. This is done by a dimension 
count, but we have no reason to expect that the relevant incidence correspondence is irreducible. Therefore the argument needs some dressing up!

Let $\left\{P_{1}, \ldots, P_{\rho}\right\}$ be a set of $\rho$ points on $Q$. We say that this set is special if, for some $\mu \leqslant \frac{1}{2} \rho$, it contains a subset of $2 \mu$ points that belong to an irreducible curve of degree $\mu$ and geometric genus zero lying on $Q$. By Lemma 2.5, there is a Zariski-open subset $U \subset(Q)^{\rho}$ such that if $\left(P_{1}, \ldots, P_{\rho}\right) \in U$ then $\left\{P_{1}, \ldots, P_{\rho}\right\}$ is non-special.

Let $\mathscr{B}_{Q}=U \times\left(G_{1,3}\right)^{2 \sigma+2}$ and consider the correspondence

$$
\mathscr{E}_{Q}=\psi_{\rho, 2 \sigma+2}^{-1}\left(\mathscr{B}_{Q}\right) \subset \mathscr{R}_{m} \times \mathscr{B}_{Q} .
$$

Finally, let $\psi=\left.\psi_{\rho, 2 \sigma+2}\right|_{\mathscr{E}_{Q}}$ and $\varphi=\left.\varphi_{\rho, 2 \sigma+2}\right|_{\mathscr{E}_{Q}}$. In order to show that the general fibre of $\psi$ is finite, it will suffice to prove that

$$
\operatorname{dim} \mathscr{E}_{Q} \leqslant \operatorname{dim} \mathscr{B}_{Q} .
$$

(As usual, $\operatorname{dim} \mathscr{E}_{Q}$ denotes the maximum of the dimensions of the irreducible components of $\mathscr{E}_{Q}$. As a matter of fact, equality holds in (14), since we know already that $C_{0} \ell^{2} \neq 0$.)

Let $\Gamma=\Gamma_{1}+\Gamma_{2}$ be any element of $\mathscr{R}_{m}$, with $\Gamma_{1} \cap Q$ finite and $\Gamma_{2} \subset Q$. Write $m_{i}=\operatorname{deg} \Gamma_{i}(i=1,2)$. If $m_{2}=0$, then $\Gamma \cap Q$ is finite; therefore the fibre of $\varphi$ above $\Gamma$ has dimension $3(2 \sigma+2)$. If, on the other hand, $m_{2}>0$, then a set of $\rho$ points in $\Gamma \cap Q$ consists of $\rho_{1} \leqslant \rho$ points in $\Gamma_{1} \cap Q$, together with $\rho_{2}=\rho-\rho_{1}$ points on $\Gamma_{2}$. By definition this set is non-special only if $\rho_{2} \leqslant 2 m_{2}-1$. (Indeed, $\Gamma_{2}$ is a union of irreducible curves of genus zero, as we saw in the proof of Lemma 2.6.) Since the first $\rho_{1}$ points vary in a finite set and the other $\rho_{2}$ points are chosen on a curve, we see that the fibre of $\varphi$ above $\Gamma$ has dimension at most $\left(2 m_{2}-1\right)+3(2 \sigma+2)$. Moreover, by Lemmas 2.4 and 2.5 , the dimension of the family of such 1-cycles $\Gamma=\Gamma_{1}+\Gamma_{2}$ does not exceed $4 m_{1}+\left(2 m_{2}-1\right)$. Thus the dimension of $\mathscr{E}_{Q}$ is bounded by the maximum of $4 m+6 \sigma+6$ (which corresponds to the case where $m_{2}=0$ ) and of the numbers $\left(4 m_{1}+2 m_{2}-1\right)+\left(2 m_{2}-1+6 \sigma+6\right)=4 m-2+6 \sigma+6$. Hence, by (13), we have

$$
\operatorname{dim} \mathscr{E}_{Q} \leqslant 4 m+6 \sigma+6=2 \rho+4(2 \sigma+2)=\operatorname{dim} \mathscr{B}_{Q} .
$$

COROllary 3.2. The set of curves $\Gamma \in \mathscr{R}_{m}$ that satisfy either $C \ell^{2}$ or $C_{0} \ell^{2}$ is finite and non-empty. This assertion holds in particular for $\ell^{4 m}$.

Proof. For $C=P^{2 m-1}$, this is Lemma 2.6. Then the general case is proved inductively by means of Lemma 3.1: it suffices to show that $C P$ is non zero; but, if $C=P^{\rho} \ell^{2 \sigma}$, then $C P=\left(P^{\rho+1} \ell^{2 \sigma-2}\right) \ell^{2}$.

Corollary 3.2.1. The set of curves $\Gamma \in \mathscr{R}_{m}$ that satisfy either $C_{0} P$ or $C_{0} P_{0}$ is finite. It is empty only if $m=2$ and $C=P^{3}$.

Proof. If $C=P^{2 m-1}$, this is Lemma 2.7. Otherwise, $\sigma \geqslant 1$ and the result is contained in Corollary 3.2 .

Corollary 3.2.2. Any curve $\Gamma \in \mathscr{R}_{m}$ satisfying $C_{0} \ell^{2}$ or $C_{0} P_{0}$ is irreducible.

Proof. This follows from Corollaries 3.2 and 3.2.1 as in Corollary 2.7.2.

Corollary 3.3. The set of curves $\Gamma \in \mathscr{R}_{m}$ that satisfy $C$ (or $C_{0}$ ) and meet two prescribed lines $L_{1}, L_{2}$, which are generic apart from their intersecting in one generic point $R \in \mathbb{P}^{3}$, is finite and non-empty. 
Proof. Let $S$ be the union of all the curves $\Gamma \in \mathscr{R}_{m}$ that satisfy $C$ (respectively $C_{0}$ ) and meet $L_{1}$. By Corollary 3.2 and an argument already used in the proof of Lemma 3.1, we see that $\operatorname{dim} S=2$ : indeed $\operatorname{dim} S>1$ because $C \ell^{2} \neq 0$; and $\operatorname{dim} S<3$ because $C_{0} \ell^{2}$ is finite. If $L_{2}^{\prime}$ is a generic line in $\mathbb{P}^{3}$ (over the field $K=k\left(C, L_{1}\right)$ over which the conditions are defined), then $L_{2}^{\prime}$ meets each two-dimensional component $S_{i}$ of $S$ in a generic point of $S_{i}$ (over $K$ ). Therefore, by Corollary 3.2, there pass only finitely many generating curves of $S_{i}$ through such a generic point. For $L_{2}$ generic through $R$ (over $K(R)$ ), the intersections of $L_{2}$ with $S$ therefore correspond to finitely many curves meeting $L_{2}$. This includes the set of curves satisfying $C P$, respectively $C_{0} P$, which is finite by Corollary 3.2.1.

We can at last attack the more difficult question of multiplicities. First we note that the condition $C P$ is a specialization of $C \ell^{2}$, in the following sense: let $\mathscr{E}_{\rho, 2 \sigma+2} \subset \mathscr{R}_{m} \times \mathscr{B}_{\rho, 2 \sigma+2}$ be the irreducible correspondence associated $(\S 2 \mathrm{~A})$ with the condition $C \ell^{2}$. A generic point of $\mathscr{E}$ lies above a generic point $\left(C, L_{1}^{\prime}, L_{2}^{\prime}\right) \in \mathscr{B}$. If we specialize the two lines in $G_{1,3} \times G_{1,3}$ so that their specializations $L_{1}, L_{2}$ meet in one generic point $R \in \mathbb{P}^{3}$ (over $k(C)$ ), then the fibre of $\psi$ above $\left(C, L_{1}, L_{2}\right)$ contains all the curves $\Gamma \in \mathscr{R}_{m}$ that satisfy $C$ and pass through $R$. Of course, it contains also some other curves, which satisfy $C$ and meet $L_{1}$ and $L_{2}$ without passing through $R$. By Corollary 3.3, this fibre is finite. Moreover, since $\mathscr{E}$ is irreducible and $\mathscr{B}$ is normal, the principle of conservation of number applies. Hence we have

COROLlary 3.4. The following inequalities hold:

$$
P^{2 m} \leqslant P^{2 m-1} \ell^{2} \leqslant \ldots \leqslant P \ell^{4 m-2} \leqslant \ell^{4 m} .
$$

Another consequence of this remark is that the curves satisfying $P^{2 m}$, i.e. belonging to a given generic fibre of $\psi_{2 m, 0}$, occur together in some finite fibre of every morphism $\psi_{\alpha, \beta}$, including $\psi_{0,4 m}$, which is associated with the condition $\ell^{4 m}$. By Corollaries 3.2 and 3.2.1, we know that the general fibre of every map $\psi_{\alpha, \beta}$ (with $\beta>0$ if $m=2$ ) is finite and non-empty, even above the specializations $C \rightarrow C_{0}$. Moreover, since the correspondence $\mathscr{E}_{0,4 m}$ is irreducible and the base $\mathscr{B}_{0,4 m}$ is normal, every finite fibre is a specialization of the general fibre of $\psi_{0,4 m}$ (cf. [4, Chapter 11, §7]). We can therefore view the correspondence $\mathscr{E}_{0,4 m}$ as being the most general. A priori, a curve $\Gamma \in \mathscr{R}_{m}$ passing through $2 m$ generic points may have to be counted with different multiplicities in the various correspondences $\mathscr{E}_{\alpha, \beta}$. Lemma 3.6 below asserts that this is not the case: for every specialization of the fibres of $\psi_{0,4 m}$, the curve $\Gamma$ is the specialization of just one curve in the general fibre (i.e. satisfying the general condition $\ell^{4 m}$ ). This transversality statement is established by considering successively all correspondences $\mathscr{E}_{\rho, 2 \sigma+2}$ for $\sigma=0, \ldots, 2 m-1$. We begin by introducing some further notation.

We consider a fixed set of $\rho$ generic points on $Q$ and $2 \sigma$ generic lines in $\mathbb{P}^{3}$, which define the condition $C_{0}$. Let $\mathscr{A} \subset \mathscr{R}_{m}$ be the subvariety consisting of all the curves which satisfy $C_{0}$. Then $\operatorname{dim} \mathscr{A} \leqslant 2$; indeed, suppose $\mathscr{A}$ has a component $\mathscr{A}^{\prime}$ with dimension at least 3 . Let $\mathscr{E}^{\prime} \subset \mathscr{A}^{\prime} \times \mathbb{P}^{3}$ be the relevant incidence correspondence, which is irreducible as in Lemma 2.0. As the first projection $\varphi^{\prime}$ is surjective with onedimensional fibres, $\mathscr{E}^{\prime}$ has dimension at least 4. This contradicts Corollary 3.2.1, according to which the general fibre of the second projection $\psi^{\prime}$ is finite and nonempty. It follows that

$$
\operatorname{dim} \mathscr{A}=2 .
$$

For if $\operatorname{dim} \mathscr{A} \leqslant 1$, then the union of these curves would span at most a surface and $C_{0} \ell^{2}$ would be zero, contrary to the assertion of Corollary 3.2. 
Let $\mathscr{A}_{0} \subset \mathscr{A}$ be the open subset consisting of the curves $\Gamma \in \mathscr{A}$, no component of which lies on $Q$. By Corollary 3.2.2, $\mathscr{A}$ contains some irreducible curves. Moreover, it is clear (Lemma 2.5) that only finitely many irreducible curves satisfying $C_{0}$ lie on $Q$. Therefore $\mathscr{A}_{0} \neq \varnothing$ and we can see as above that

$$
\operatorname{dim} \mathscr{A}_{0}=2 .
$$

We have not ruled out the possibility that $\mathscr{A}_{0}$ may be reducible, with some component of dimension smaller than 2 .

Let $\mathscr{B}_{0}=\left\{\left(C_{0}, L_{1}, L_{2}\right) \in \mathscr{B}_{\rho, 2 \sigma+2}\right\}$; since $C_{0}$ is fixed, $\mathscr{B}_{0}$ is obviously isomorphic to $G_{1,3} \times G_{1,3}$. We define $\mathscr{E}_{0} \subset \mathscr{A}_{0} \times \mathscr{B}_{0}$ to be the incidence correspondence

$$
\mathscr{E}_{0}=\mathscr{E}_{\rho, 2 \sigma+2} \cap\left(\mathscr{A}_{0} \times \mathscr{B}_{0}\right) \text {. }
$$

Let $\varphi_{0}=\left.\varphi_{\rho, 2 \sigma+2}\right|_{\delta_{0}}$ and $\psi_{0}=\left.\psi_{\rho, 2 \sigma+2}\right|_{\delta_{0}}$. Now let

$\mathscr{D}=\left\{\left(C_{0}, L_{1}, L_{2}\right) \in \mathscr{B}_{0} \mid L_{1}\right.$ (respectively $\left.L_{2}\right)$ is a member of the

first (respectively second) ruling of $Q$ \}.

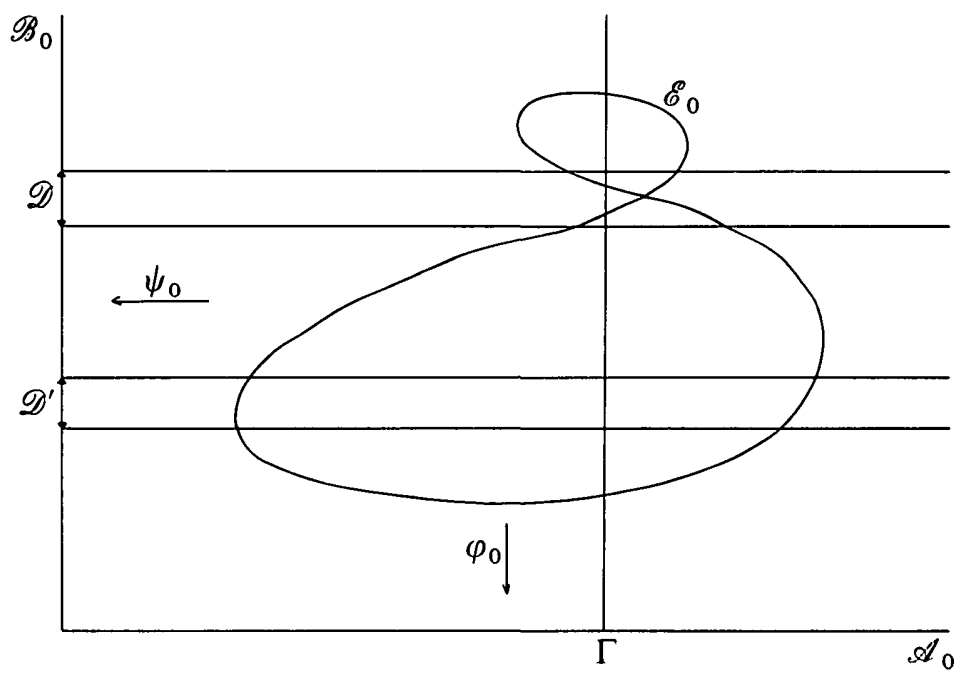

FIG. 1.

Then we have the following lemma.

Lemma 3.5. If $d$ is a generic point of $\mathscr{D}$, then $\psi_{0}^{-1}(d)$ is finite. It is empty only if $m=2$ and $C=P^{3}$.

Proof. For any $\Gamma \in \mathscr{A}_{0}$, the set $\varphi_{0}{ }^{-1}(\Gamma) \cap \psi_{0}{ }^{-1}(\mathscr{D})$ is finite, since $\Gamma$ meets $Q$ in finitely many points. Hence, by (16), every irreducible component of $\psi_{0}^{-1}(\mathscr{D})$ has dimension at most 2. Since $\operatorname{dim} \mathscr{D}=2$, this implies that $\psi_{0}^{-1}(d)$ is finite for $d$ generic in $\mathscr{D}$.

Now, by Corollary 3.2.1, the set of curves satisfying $C_{0} P_{0}$ is not empty, unless $m=2$ and $C=P^{3}$. Moreover, as we saw in the discussion of (16), this set contains some irreducible curves $\Gamma \in \mathscr{A}_{0}$. Hence $\psi_{0}{ }^{-1}(d)$ is non-empty for $d \in \mathscr{D}$ generic, except when $m=2$ and $C=P^{3}$.

Lemma 3.6. For every specialization of the fibres of $\psi_{\rho .2 \sigma+2}$, every curve $\Gamma \in \mathscr{R}_{m}$ that satisfies $C P$ is the specialization of a unique curve satisfying $C \ell^{2}$. In other words, 
given $R$ generic in $\mathbb{P}^{3}$ and two generic lines $L_{1}, L_{2}$ intersecting at $R$, the fibre of $\psi_{\rho, 2 \sigma+2}$ above $\left(C, L_{1}, L_{2}\right)$ is transversal to $\mathscr{E}_{\rho, 2 \sigma+2}$ at $\left(\Gamma, C, L_{1}, L_{2}\right)$. The same statement holds for $C_{0} \ell^{2}, C_{0} P$, and $C_{0} P_{0}$.

Proof. We give the argument for the condition $C_{0} P_{0}$, from which all the other cases derive. If $\Gamma \in \mathscr{R}_{m}$ satisfies $C_{0} P_{0}$, we know (Corollary 3.2.2 and Lemma 2.5) that $\Gamma$ is irreducible and does not lie on $Q$, whence $\Gamma \in \mathscr{A}_{0}$. Moreover, $\Gamma$ lies above a generic point $d \in \mathscr{D}$. From Lemma 3.5, we know that $\psi_{0}^{-1}(d)$ is finite. Therefore all we have to prove is that the fibre of $\psi_{0}$ is transversal to $\mathscr{E}_{0}$ at $(\Gamma, d) \in \mathscr{E}_{0}$.

Let $\mathscr{A}^{\prime}$ be any irreducible component of $\mathscr{A}_{0}$. If $\operatorname{dim} \mathscr{A}^{\prime} \leqslant 1$, then

$$
\mathscr{E}^{\prime}=\varphi_{0}^{-1}\left(\mathscr{A}^{\prime}\right) \cap \psi_{0}^{-1}(\mathscr{D})
$$

has dimension less than 2. Hence $\psi_{0}\left(\mathscr{E}^{\prime}\right)$ contains no generic point of $\mathscr{D}$, and we can forget about any such component. We shall therefore assume that $\operatorname{dim} \mathscr{A}^{\prime}=2$ and that $\psi_{0}\left(\mathscr{E}^{\prime}\right)$ contains a generic point of $\mathscr{D}$. Then a generic curve $\Gamma \in \mathscr{A}^{\prime}$ meets $Q$ in $2 m$ distinct points, and thus meets $Q$ on $2 m$ distinct lines of the first ruling of $Q$, and $2 m$ distinct lines of the second ruling. Hence

$$
\operatorname{card}\left(\varphi_{0}^{-1}(\Gamma) \cap \psi_{0}^{-1}(\mathscr{D})\right)=4 m^{2} .
$$

The transversality assertion will follow from (17) by Schubert theory. To begin with, let $\mathscr{D}^{\prime}$ be a generic subvariety of $\mathscr{B}_{0} \approx G_{1,3} \times G_{1,3}$ in the numerical equivalence class of $\mathscr{D}$. Then we also have

$$
\operatorname{card}\left(\varphi_{0}{ }^{-1}(\Gamma) \cap \psi_{0}^{-1}\left(\mathscr{D}^{\prime}\right)\right)=4 m^{2} .
$$

In fact, if we identify $G_{1,3}$ with the Klein quadric (cf. $[12$, Chapter $\left.10, \S 2]\right) \Omega \subset \mathbb{P}^{5}$, a ruling of $Q$ is represented by a conic on $\Omega$. The complex of secants of $\Gamma \in \mathscr{R}_{m}$ is represented by a divisor $D_{m}$, which is the complete intersection of $\Omega$ with a hypersurface $G_{m}$ of degree $m$ in $\mathbb{P}^{5}$. Now a general conic on $\Omega$ cannot meet $G_{m}$ in more than $2 m$ distinct points, whence (18) holds, since equality holds for the specialization $\mathscr{D}$ of $\mathscr{D}^{\prime}$, by virtue of (17).

Now let $\mathscr{E}_{0}^{\prime \prime}=\varphi_{0}{ }^{-1}\left(\mathscr{A}^{\prime}\right)$. Since $\mathscr{A}^{\prime}$ contains the Chow point of some irreducible curve (Lemma 3.2.2), one sees as in Lemma 2.1 that $\mathscr{E}_{0}^{\prime}$ is irreducible. Moreover, $\psi_{0}\left(\mathscr{E}_{0}^{\prime}\right)$ contains a generic point of $\mathscr{B}_{0}$ : indeed $\psi_{0}\left(\mathscr{E}_{0}^{\prime}\right)$ contains $\psi_{0}\left(\mathscr{E}^{\prime}\right)$, which we assume to contain a generic point $d$ of $\mathscr{D}$; and $\psi_{0}^{-1}(d)$ is finite, by Lemma 3.5; hence the general fibre of $\psi_{0}$ is finite and non-empty, since $\operatorname{dim} \mathscr{E}_{0}^{\prime}=8=\operatorname{dim} \mathscr{B}_{0}$.

Finally, since a generic point $d^{\prime}$ of $\mathscr{D}^{\prime}$ is also a generic point of $\mathscr{B}_{0}$, the fibre $\psi_{0}^{-1}\left(d^{\prime}\right) \cap \mathscr{E}_{0}^{\prime}$ above $d^{\prime}$ is transversal to $\mathscr{E}_{0}^{\prime}$ (the characteristic is equal to zero). Therefore, for generic $\Gamma \in \mathscr{A}^{\prime}$, the intersection

$$
\psi_{0}{ }^{-1}\left(\mathscr{D}^{\prime}\right) \cap\left(\{\Gamma\} \times \mathscr{B}_{0}\right)
$$

is defined (in $\mathscr{A}_{0} \times \mathscr{B}_{0}$ ) and consists of distinct points with multiplicity one:

$$
\operatorname{deg}\left\{\left(\{\Gamma\} \times \mathscr{B}_{0}\right) \cdot\left(\mathscr{E}_{0} \cdot\left(\mathscr{A}_{0} \times \mathscr{D}^{\prime}\right)\right)\right\}=\operatorname{card}\left(\varphi_{0}{ }^{-1}(\Gamma) \cap \psi_{0}{ }^{-1}\left(\mathscr{D}^{\prime}\right)\right) .
$$

This can also be seen by applying [5, Theorem 2], with $X=\mathscr{B}_{0}, Y=\mathscr{D}$ (or $\mathscr{D}^{\prime}$ ), and $Z$ as the regular locus of $\varphi_{0}^{-1}(\Gamma)$.

On the other hand, $\mathscr{D}^{\prime}$ is numerically equivalent to $\mathscr{D}$ (and $\mathscr{B}_{0}$ is smooth, as well as $\left.\Gamma \in \mathscr{A}^{\prime}\right)$; hence, by (17), (18), and (19), we have

$$
\operatorname{deg}\left\{\left(\{\Gamma\} \times \mathscr{B}_{0}\right) \cdot\left(\mathscr{E}_{0} \cdot\left(\mathscr{A}_{0} \times \mathscr{D}\right)\right)\right\}=\operatorname{card}\left(\varphi_{0}^{-1}(\Gamma) \cap \psi_{0}^{-1}(\mathscr{D})\right) .
$$


It follows from (20) that the fibre of $\psi_{0}$ is transversal to $\mathscr{E}_{0}^{\prime}$ at all points $(\Gamma, d) \in \varphi_{0}{ }^{-1}\left(\mathscr{A}^{\prime}\right) \cap \psi_{0}{ }^{-1}(d)$. Otherwise, since $d$ is generic in $\mathscr{D}$, the intersection $\mathscr{E}_{0} .\left(\mathscr{A}_{0} \times \mathscr{D}\right)$ would have a multiple component (by [16, Chapter $6, \S 2$, Theorem 6]: apply the converse to $A=\mathscr{A}^{\prime} \times \mathscr{D}, B=\mathscr{E}_{0}^{\prime}, U=\mathscr{A}^{\prime} \times \mathscr{B}_{0}, P=(\Gamma, d)$ ) and equality could not hold in (20).

The lesson of this proof is that the transversality of the fibres of $\psi_{0}$ over a subvariety of $\mathscr{B}_{0}$ can be ascertained by watching how the fibres of $\varphi_{0}$ behave when we move that subvariety.

COROLlaRy 3.7. The number of distinct curves $\Gamma \in \mathscr{R}_{m}$ passing through $2 m$ points is the same whether the points are generic in $\mathbb{P}^{3}$ or only generic on $Q$. The corresponding result holds also for $P^{2 m-1} \ell^{2}$ and all other conditions $P^{\alpha} \ell^{2 \beta}$ with $\alpha+\beta=2 m$.

Proof. This is proved inductively, by means of Lemma 3.6. Every curve satisfying $C_{0} P$ is the specialization of just one curve satisfying $C_{0} \ell^{2}$; and the same statement holds for $C_{0} P_{0}$. Hence distinct curves satisfying $C_{0} P$ specialize to distinct curves satisfying $C_{0} P_{0}$. Therefore $C_{0} P \leqslant C_{0} P_{0}$.

Moreover, if a curve $\Gamma$ satisfies $C_{0}$ and meets two lines $L_{1}, L_{2}$ intersecting in one generic point $R \in \mathbb{P}^{3}$, then $\Gamma$ cannot specialize to a curve satisfying $C_{0} P_{0}$ unless $R \in \Gamma$. Otherwise, the degree of the fibres of $\psi_{\rho+1,2 \sigma}$ would grow as $R \in \mathbb{P}^{3}$ specializes to $R_{0} \in Q$, and this would contradict the principle of conservation of number. Therefore $C_{0} P=C_{0} P_{0}$. Applying this result inductively, we see that each curve satisfying $P_{0}^{2 m}$ (respectively $P^{2 m}$ ) is the specialization of just one curve satisfying $\ell^{4 m}$, so that $P_{0}^{2 m}=P^{2 m}$, and similarly for the other conditions.

Finally, we observe that the precise meaning of 'genericity' for the choice of $2 \mathrm{~m}$ points on $Q$ in Corollary 3.7 is much less clear than in $\S 2$. It can be shown, with the methods of $\S 3 \mathrm{~B}$, that it is not enough to require that this set of points should be nonspecial, as defined in the proof of Lemma 3.1. In particular, one must avoid certain pinch-points of the surface spanned by the curves satisfying $P_{0}^{2 m-1} \ell$.

\section{B. A conjecture}

In the rest of this section we shall consider the two specializations of the condition $P^{2 m-1} \ell^{2}$ which were described in $\S 2 \mathrm{~B}$. Let $\mathscr{A}$ be the subvariety of $\mathscr{R}_{m}$ corresponding to the condition $C_{0}=P_{0}^{2 m-1}$, as defined in the discussion preceding Lemma 3.5. Further, let

$$
\mathscr{B}_{0}=\left\{\left(C_{0}, L_{1}, L_{2}\right) \in \mathscr{B}_{2 m-1,2}\right\} \approx G_{1,3} \times G_{1,3}
$$

and consider the induced correspondence $\mathscr{E} \subset \mathscr{A} \times \mathscr{B}_{0}$, with the two projections $\varphi: \mathscr{E} \rightarrow \mathscr{A}$ and $\psi: \mathscr{E} \rightarrow \mathscr{B}_{0}$. Let $L_{1}$ and $L_{1}^{\prime}$ be two generic lines of the first ruling of $Q$; let $L_{2}$ be a generic line of the second ruling. By Lemma 2.6, we may define

$$
U=\operatorname{deg} \psi^{*}\left(L_{1}, L_{1}^{\prime}\right), \quad V=\operatorname{deg}_{0} \psi^{*}\left(L_{1}, L_{2}\right),
$$

where the notation $\operatorname{deg}_{0}$ means that we count in only the curves $\Gamma$ that do not pass through $L_{1} \cap L_{2}$. It follows from Corollary 3.7 that

and

$$
P^{2 m-1} \ell^{2}=U
$$

$$
P^{2 m}=U-V .
$$


To prove Theorems 1 and 2 (stated in $\S 1 \mathrm{~A}$ ), we are therefore reduced to determining $U$ and $V$, which are certain (weighted) numbers of curves lying on $Q$ (because of the Bézout theorem). It is a consequence of Lemma 2.6 that both $U$ and $V$ are certain combinations of the $\Delta_{\mu, v}$ (defined in $\S 1 \mathrm{~A}$ ), with coefficients to be determined.

It is generally asserted in the classical literature that 'any degenerate cubic which meets $k$ times a line which it is only required to meet once will be a $k$-ple solution of the problem, and must be so reckoned in the enumeration' [14, p. 297]. If this is true in general, the coefficients of the $\Delta_{\mu, \nu}$ are quite easy to determine. Indeed, a curve of type $(\mu, v)$ meets $L_{1}$, and also $L_{1}^{\prime}$, in $v$ points (instead of one); hence it is counted with multiplicity $v^{2}$ in $\psi^{*}\left(L_{1}, L_{1}^{\prime}\right)$. Similarly, it occurs with multiplicity $\mu v$ in $\psi^{*}\left(L_{1}, L_{2}\right)$. Hence

$$
U=\sum_{\substack{v=1 \\ \mu+v=m}}^{m} v^{2} \Delta_{\mu, v},
$$

which is Theorem 2, and

$$
V=\sum_{\substack{\mu, v \geqslant 1 \\ \mu+v=m}} \mu \nu \Delta_{\mu, v} .
$$

Therefore

$$
\begin{aligned}
P^{2 m}=U-V & =\sum_{\substack{\mu, v \geqslant 0 \\
\mu+v=m}}\left(v^{2}-\mu v\right) \Delta_{\mu, v} \\
& =\sum_{\substack{0 \leq \mu<v \\
\mu+\nu=m}}\left[\left(\mu^{2}-\mu v\right)+\left(v^{2}-\mu v\right)\right] \Delta_{\mu, v} \\
& =\sum_{\substack{0 \leq \mu<v \\
\mu+\nu=m}}(\mu-v)^{2} \Delta_{\mu, v} .
\end{aligned}
$$

Taking Lemma 1.2 into account, we therefore obtain

$$
P^{2 m}=(m-2)^{2}+\sum_{2 \leqslant \mu<\frac{1}{2} m}(m-2 \mu)^{2} \Delta_{\mu, m-\mu},
$$

which is Theorem 1.

All we have to do is to justify this way of counting multiplicities. We shall make an attempt by studying the surface $S$ spanned by the curves $\Gamma \in \mathscr{R}_{m}$ that satisfy $C_{0}$ and meet some line $L \subset \mathbb{P}^{3}$. This method was used extensively by $R$. Sturm [13] for obtaining many difficult numbers in the enumerative theory of twisted cubics. However, we shall not succeed without making a certain assumption, which relates to the structure of the Chow variety. We begin this discussion with an easy lemma.

Lemma 3.8. $L_{0}$ being a generic line of $Q$, let $S_{0}$ be the union of all the curves $\Gamma \in \mathscr{R}_{m}$ that satisfy $C_{0}$ and meet $L_{0}$. Then $S_{0}$ is a surface. Suppose $S_{0}$ is irreducible. Then there is a positive integer $\lambda_{0}$ such that the (weighted) number of curves in the family that pass through any normal point of $S_{0}$ is equal to $\lambda_{0}$ (or, exceptionally, infinite). Suppose $\lambda_{0}=1$. Then the line $L_{0}$ is $P^{2 m}$-fold on $S_{0}$, and the degree of $S_{0}$ is equal to $\overline{P^{2 m-1} \ell^{2}}$.

Proof. By an argument resembling that used in Lemma 3.5, we see that the set of curves $\Gamma \in \mathscr{R}_{m}$ satisfying $C_{0}$ and meeting $L_{0}$ is an algebraic variety $\mathscr{S}$ of dimension 1 . 
Hence $S_{0}$ is a surface. Consider the incidence correspondence

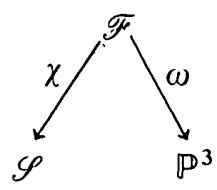

If we knew that $\mathscr{S}$ was irreducible, the same property would hold for $\mathscr{F}$ (cf. Lemma 2.0 ), and hence also for its image $S_{0}=\omega(\mathscr{F}) \subset \mathbb{P}^{3}$. As it stands, we are assuming that $S_{0}$ is irreducible. (At any rate, even without this assumption, it is clear from the Bézout theorem that $S_{0} \not Q$, since only finitely many curves of $Q$ satisfy $C_{0}$.)

Let $R$ be any normal point of $S_{0}$. By the principle of conservation of number [8, Chapter $2, \S 5.3$, Theorems 6 and 7], there is a definite number $\lambda_{0}$ of curves through $R$, counted with the multiplicities appearing in the corresponding fibre of $\omega$, unless this fibre is (exceptionally) infinite. We are assuming that $\lambda_{0}=1$. Now a generic line $L^{\prime} \subset \mathbb{P}^{3}$ meets $S_{0}$ transversally in $\operatorname{deg}\left(S_{0}\right)$ simple points, corresponding to $\lambda_{0} \cdot \operatorname{deg}\left(S_{0}\right)=\operatorname{deg}\left(S_{0}\right)$ curves $\Gamma \in \mathscr{S}$. These are precisely the curves $\Gamma \in \mathscr{R}_{m}$ that satisfy the condition $C_{0}$ and meet $L_{0}$ and $L^{\prime}$. Hence, by Lemma 3.6, $\operatorname{deg}\left(S_{0}\right)=P^{2 m-1} \ell^{2}$. (Note that in the proof of Lemma 3.6, we used the lines of $Q$; hence our assertion is justified, even though $L_{0}$ lies on $Q$ instead of being generic in $\mathbb{P}^{3}$. The only thing that matters is that the curves $\Gamma$ which satisfy the condition do not lie on $Q$ (i.e. their Chow point is in $\mathscr{A}_{0} \subset \mathscr{A}$ with $\mathscr{A}_{0} \neq \mathscr{A}$ ).)

Similarly, if we choose $L^{\prime} \subset \mathbb{P}^{3}$ generic amongst the lines passing through a generic point $R$ of $L_{0}$, then $L^{\prime}$ meets $S_{0} \backslash\{R\}$ transversally in $V$ points. Hence $L^{\prime}$ meets $S_{0}$ with multiplicity $\operatorname{deg}\left(S_{0}\right)-V=U-V=P^{2 m}$ at $R$. It follows that the line $L_{0}$ is exactly $P^{2 m}$-fold on $S_{0}$.

It is probably true that $S_{0}$ is irreducible, but this assumption is not important. If we do not want to make it, then each component of $S_{0}$ comes equipped with a number $\lambda_{i}$, and the lemma remains true if we suppose that all the $\lambda_{i}$ are equal to 1 . In fact, even this last assumption is relatively unimportant for what follows; without it, Lemma 3.8 is more difficult to enunciate, but it has the same consequences. For simplicity, however, we shall assume from now on that $S_{0}$ is irreducible; and also that $\lambda_{0}=1$, since anyway this is the easiest corollary of the conjecture we shall introduce below.

Lemma 3.9. Let $\Gamma_{\mu, v}$ be one of the $\Delta_{\mu, v}$ curves of type $(\mu, v)$ lying on $Q$ which satisfy $C_{0}$. Let $L$ be a line in $\mathbb{P}^{3}$ that meets $\Gamma_{\mu, \nu}$ in one point $R$ and is otherwise generic. Consider the union $S$ of all the curves $\Gamma \in \mathscr{R}_{m}$ that satisfy $C_{0}$ and meet $L$. Then $S$ is a surface. Suppose $S$ is irreducible. Then, through any normal point of $S$, there passes precisely one curve in the family (or infinitely many, in exceptional cases). Furthermore, if $L_{0}$ belongs (say) to the first ruling of $Q$ (and $\lambda_{0}=1$ ), then the following relation holds between the multiplicities of $\Gamma_{\mu, \nu}$ on $S$ and on $S_{0}$ :

$$
\text { mult }_{s_{0}} \Gamma_{\mu, v}=v \cdot \text { mult }_{S} \Gamma_{\mu, v} .
$$

Proof. As in the preceding lemma, the number of curves $\Gamma$ passing through a normal point of $S$, when finite, is a well-defined integer $\lambda$. But in the present case we can prove that $\lambda=1$. Indeed, let $T$ be a generic point of $\mathbb{P}^{3}$ and consider the finitely many curves through $T$ that satisfy $C_{0}$ (Lemma 2.7). Clearly, we can choose $R$ on $\Gamma_{\mu, v}$ and a line $L$ through $R$ in such a way that $L$ meets one of these curves and not the others. Then, 
through $T \in S$, there is a unique curve satisfying $C_{0}$ and meeting $L$, and it occurs with multiplicity 1 (Corollary 3.7). Hence $\lambda=1$.

Now we have two ways of computing the multiplicity ( $\rho$ say), with which $\Gamma_{\mu, v}$ occurs in the set of curves satisfying the condition $C_{0}$ and meeting $L$ and $L_{0}$. Indeed, the intersection multiplicity of $L$ with $S_{0}$ at $R$ is equal to the multiplicity of $\Gamma_{\mu, v}$ on $S_{0}$; hence $\rho=\lambda_{0}$. mult ${ }_{S_{0}} \Gamma_{\mu, v}$. On the other hand, $L_{0}$, being generic on $Q$, meets $S$ transversally at each of its $v$ intersection points with $\Gamma_{\mu, v}$. Since $\lambda=1$, it follows that $\rho=v \cdot$ mult $_{s} \Gamma_{\mu, v}$. Therefore,

$$
\lambda_{0} \cdot \text { mult }_{S_{0}} \Gamma_{\mu, v}=v \cdot \text { mult }_{S} \Gamma_{\mu, v} .
$$

This is the required formula, since we have made the assumption that $\lambda_{0}=1$.

We now introduce the following conjecture.

CONJECTURE $(*)$. The curve $\Gamma_{\mu, v}$ is simple on $S$.

Indeed it seems reasonable to expect that mult $\Gamma_{\mu, v}$ is a (symmetric) function of $\mu$ and $v$ only. To assume mult $\Gamma_{\mu, v}=1$ is equivalent to saying that $\Gamma_{\mu, v}$ occurs with multiplicity 1 among all the curves $\Gamma \in \mathscr{R}_{m}$ that satisfy $C_{0}$ and meet two general secants $L$ and $L^{\prime}$ of $\Gamma_{\mu, v}$ in $\mathbb{P}^{3}$. We know from Corollary 3.7 that it is not too restrictive to impose $2 m-1$ points lying on $Q$. Hence the main difficulty is that the curve $\Gamma_{\mu, \nu}$ itself lies on $Q$; therefore its Chow point might be very special. It is true that, when $\Gamma_{\mu, v}$ is smooth (i.e. if $\mu$ or $v$ is equal to 1 ), its Chow point is also smooth, since the normal bundle verifies the Kodaira-Spencer condition $h^{1}(\mathcal{N})=0$. In this case, it may be possible to use an argument similar to the one used in Lemma 3.6 (with some wellchosen class of subvarieties $\mathscr{D}$ ), in order to prove that $\operatorname{mult}_{S} \Gamma_{m-1,1}=1$. For other values of $\mu$ and $v$, the conjecture seems difficult to prove; and it would almost certainly be false if the Chow point of $\Gamma_{\mu, v}$ turned out to be singular on $\mathscr{R}_{m}$.

It is interesting to note that the simplest case of this conjecture already implies that $\lambda_{0}=1$ (if we assume that $S_{0}$ is irreducible). Indeed, it suffices to apply (27) to the curve $\Gamma_{m-1,1}$ (which always exists); we get

Hence $\lambda_{0}=1$.

$$
\lambda_{0} \text {. } \text { unit }_{S_{0}} \Gamma_{m-1,1}=\text { mult }_{s} \Gamma_{m-1,1}=1 .
$$

CoROLlaRY $3.9 .1^{*}$. mult $_{S_{0}} \Gamma_{\mu, v}=v$.

Proof. This is an immediate consequence of Lemma 3.9 and the conjecture.

COROLlaRY $3.10^{*}$. (24) and (25) are valid.

Proof. By Lemma 3.8, $U$ is the degree of $S_{0}$. Therefore it is enough to compute the intersection number of $S_{0}$ with a generic line $L_{0}^{\prime} \subset Q$ belonging to the same ruling as $L_{0}$. Now the curves $\Gamma \in \mathscr{R}_{m}$ that satisfy $C_{0}$ and meet $L_{0}$ and $L_{0}^{\prime}$ are precisely the curves $\Gamma_{\mu, v}$ of $Q$ that satisfy $C_{0}$. Moreover, $L_{0}^{\prime}$ meets $\Gamma_{\mu, v}$ in $v$ points, each counting with multiplicity $v$, by Corollary 3.9.1. Hence

$$
\operatorname{deg}\left(S_{0}\right)=\sum_{\substack{v=1 \\ \mu+v=m}}^{m} v^{2} \Delta_{\mu, v}
$$

as required. (25) is proved in the same way. 


\section{Determination of $\Delta_{2,3}$}

\section{A. The Jacobian curve of the associated net}

We recall that $\Delta_{2,3}$ is the number of irreducible rational curves of type $(2,3)$ passing through nine points $P_{1}, \ldots, P_{9}$ in general position on $\mathbb{P}^{1} \times \mathbb{P}^{1}$. By Lemma 1.1, the family of curves of type $(2,3)$ has dimension 11 and genus 2 . Those passing through $P_{1}, \ldots, P_{9}$ form a two-dimensional linear system $\mathfrak{N}$ (a $\left.n e t\right)$, and we have to find the number of irreducible curves in the family with two double points somewhere on the surface. Now every such curve is characterized by its equation, of the form

$$
\Phi\left(u_{0}, u_{1} ; v_{0}, v_{1}\right)=\sum_{\substack{|\alpha=2 \\ \beta|=3}} a_{\alpha \beta} u^{\alpha} v^{\beta}=0 .
$$

(As usual, $u^{\alpha}$ denotes $u_{0}^{\alpha_{0}} u_{1}^{\alpha_{1}}$, and $|\alpha|$ is the sum $\alpha_{0}+\alpha_{1}$.) The net is described by nine linear relations between the $a_{\alpha \beta}$. Let $f, g$, and $h$ be the equations of three generators, so that a general element of $\mathfrak{N}$ is described by an equation of the form $\Phi=\lambda f+\mu g+v h=0$, for some $\lambda, \mu, v \in \mathbb{C}$. The curves $\Gamma$ through $P_{1}, \ldots, P_{9}$ are therefore in one-to-one correspondence with the points $(\lambda, \mu, v) \in \mathbb{P}^{2}$. Those having (at least) one double point on $\mathbb{P}^{1} \times \mathbb{P}^{1}$ correspond to the points of some curve $M \subset \mathbb{P}^{2}$. In $\S 4 \mathrm{~B}$ we shall characterize $M$ by determining its degree, its genus, and its class. We first have to study another curve $J \subset \mathbb{P}^{1} \times \mathbb{P}^{1}$, which is defined as the locus of double points of the curves $\Gamma$ in our net. $J$ is characterized analytically as follows: let us consider a general curve $\Gamma \in \mathfrak{N}$. We may assume that $\Gamma$ has no double points in the closed set $u_{0} v_{0}=0$. In the affine set $\mathbb{A}_{00}\left(u_{0} \neq 0, v_{0} \neq 0\right)$, in which we write $u=u_{1} / u_{0}$ and $v=v_{1} / v_{0}$, the equation of $\Gamma$ reads

$$
\Phi(u, v)=(\lambda f+\mu g+v h)(u, v)=0,
$$

where $f, g$, and $h$ are of degree 2 in $u$, and 3 in $v$. The curve $\Gamma$ has a double point at $(u, v) \in \mathbb{A}_{00}$ if and only if

$$
\left.\begin{array}{c}
\lambda f_{u}^{\prime}+\mu g_{u}^{\prime}+v h_{u}^{\prime}=0, \\
\lambda f_{v}^{\prime}+\mu g_{v}^{\prime}+v h_{v}^{\prime}=0, \\
\lambda f+\mu g+v h=0,
\end{array}\right\}
$$

where $f_{u}^{\prime}$ denotes the derivative of $f$ with respect to $u$. This system of three homogeneous linear equations has a non-trivial solution if and only if

$$
\left|\begin{array}{ccc}
f_{u}^{\prime} & g_{u}^{\prime} & h_{u}^{\prime} \\
f_{v}^{\prime} & g_{v}^{\prime} & h_{v}^{\prime} \\
f & g & h
\end{array}\right|=0 .
$$

This is the equation of a curve $J_{0} \subset A_{00}$. Its closure $J \subset \mathbb{P}^{1} \times \mathbb{P}^{1}$ is called the Jacobian curve of the net generated by $f, g$, and $h$. It is easy to see that $J$ is of type $(4,7)$, i.e. its equation is bihomogeneous, of degree 4 in $u$, and 7 in $v$. Indeed, since $f$ and $f_{v}^{\prime}$ are of degree 2 in $u$, and $f_{u}^{\prime}$ is of degree $1,(31)$ would appear to be of degree 5 in $u$. As a matter of fact, the leading coefficient is identically zero, for it comes from the leading coefficients of the polynomials in the determinant; and if we multiply the first row by $\frac{1}{2} u$, we find the same leading coefficients as in the third row. Similarly, for $v$, we find that the degree is 7 , and not 8 . One can write the coefficients of degrees 4 and 7 (in $u$ and $v$ respectively) and check that, under the genericity assumption we have made, they do not vanish (cf. also [6, formula (III, 42)]). 
Lemma 4.1. The locus of double points of the curves $\Gamma_{2,3}$ in the net $\mathfrak{N}$ determined by nine generic points $P_{1}, \ldots, P_{9}$ of $\mathbb{P}^{1} \times \mathbb{P}^{1}$ is an irreducible curve $J$, of type $(4,7)$, with exactly nine ordinary double points-one at each base-point $P_{i}$-and no other singularities.

Proof. The derivative of (31) with respect to $u$ is

$$
\left|\begin{array}{ccc}
f_{u}^{\prime \prime} & g_{u}^{\prime \prime} & h_{u}^{\prime \prime} \\
f_{v}^{\prime} & g_{v}^{\prime} & h_{v}^{\prime} \\
f & g & h
\end{array}\right|+\left|\begin{array}{ccc}
f_{u}^{\prime} & g_{u}^{\prime} & h_{u}^{\prime} \\
f_{u v}^{\prime \prime} & g_{u v}^{\prime \prime} & h_{u v}^{\prime \prime} \\
f & g & h
\end{array}\right|+\left|\begin{array}{ccc}
f_{u}^{\prime} & g_{u}^{\prime} & h_{u}^{\prime} \\
f_{v}^{\prime} & g_{v}^{\prime} & h_{v}^{\prime} \\
f_{u}^{\prime} & g_{u}^{\prime} & h_{u}^{\prime}
\end{array}\right|
$$

(where the last determinant is equal to 0 ), which obviously vanishes at the $P_{i}$, since $f=g=h=0$ there. The derivative with respect to $v$ is similar; hence the $P_{i}$ are singular on $J$. Taking second derivatives of (31), we can easily see that $J$ can have a cusp or a triple point at $P_{i}$ only if the (unique) curve of the net with a double point at $P_{i}$ has a cusp or a triple point there, or if all the curves of $\mathfrak{N}$ have a fixed tangent at $P_{\boldsymbol{i}}$. Hence the $P_{i}$ are ordinary double points of $J$, since they are assumed to form a generic set. To complete the proof, it suffices to show that $J$ has no other singularities. This will automatically imply that $J$ is irreducible, for it is easy to see that any reducible curve of type $(4,7)$ with ordinary double points at all the $P_{i}$ would also have some other singularities elsewhere. ( $J$ cannot be the union of a curve of type $(1,1)$ and one of type $(3,6)$-nine intersections-because the $P_{i}$ do not lie on a conic $\Gamma_{1,1}$.) That $J$ has no other singularities than the $P_{i}$ is an immediate consequence of the genericity of the base set, via the following classical lemma, which is easily proved analytically (see [ 2 , L.3, Chapter 2, §.21, pp. 175-176]).

LEMMA 4.1.1. $P$ is a singular point of $J$ only if one of the following conditions holds:

(i) $P$ is a base point of the net;

(ii) $P$ is at least a double point for all curves of $\mathfrak{N}$ passing through it;

(iii) $P$ is a singularity of higher order of some curve of the net;

(iv) $P$ is a cuspidal point for one of the curves, and all curves through it touch the cuspidal tangent.

\section{B. The curve of moduli}

Instead of eliminating $\lambda, \mu$, and $v$ from (30), we could equally well have eliminated $u$ and $v$, thus obtaining the equation of the curve $M \subset \mathbb{P}^{2}(\lambda, \mu, v)$, whose points represent the curves having at least one double point somewhere on $\mathbb{P}^{1} \times \mathbb{P}^{1}$. We shall not carry out the process of elimination explicitly, because all we need to know is that the system of equations (30) defines a birational correspondence $E$ between $M$ and the Jacobian curve $J$ :

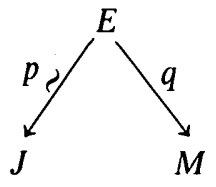

Indeed, given almost any $(\lambda, \mu, v) \in M$, there exists a point $(u, v) \in \mathbb{A}_{00}$ such that $(30)$ holds; hence $(u, v) \in J$. Besides, for almost all $(\lambda, \mu, v) \in M$, this is the only double point of the associated curve $\Gamma_{2,3}$; hence $q$ is birational. Conversely, given an arbitrary point $(u, v)$ on $J \cap \mathbb{A}_{00}$, there exists a curve with equation 
$\Phi=\lambda f+\mu g+v h=0$ for which (30) holds. And this curve is unique, by genericity (otherwise we would be in Case (ii) of Lemma 4.1.1). This implies that all the fibres of $p$ consist of one point; hence both $E$ and $M$ are irreducible. As a matter of fact, $p$ is even an isomorphism. By the Zariski main theorem, this has to be checked only above the singular points of $J$. Suppose $h=0$ is the equation of the curve of $\mathfrak{R}$ on which $P_{i}$ is double. Then $f_{u}^{\prime} g_{v}^{\prime}-f_{v}^{\prime} g_{u}^{\prime}$ does not vanish at $P_{i}$ (otherwise, all the curves of $\mathfrak{N}$ would have a fixed tangent at $P_{i}$ ) and (30) yields an explicit formulat for $p^{-1}$ in a neighbourhood of $P_{i}$ :

$$
(\lambda, \mu, v)=\left(\left|\begin{array}{ll}
-h_{u}^{\prime} & g_{u}^{\prime} \\
-h_{v}^{\prime} & g_{v}^{\prime}
\end{array}\right|, \quad\left|\begin{array}{ll}
f_{u}^{\prime} & -h_{u}^{\prime} \\
f_{v}^{\prime} & -h_{v}^{\prime}
\end{array}\right|, \quad\left|\begin{array}{ll}
f_{u}^{\prime} & g_{u}^{\prime} \\
f_{v}^{\prime} & g_{v}^{\prime}
\end{array}\right|\right) .
$$

Hence $p$ is an isomorphism, as asserted.

LeMma 4.2. Let $S \in J$ and $T=q \circ p^{-1}(S) \in M$. Suppose $S$ is not one of the base points $P_{i}$. Then the set of curves $\Gamma \in \mathfrak{N}$ passing through $S$ is mapped (via (29)) into a line $\ell \subset \mathbb{P}^{2}$, which is tangent to a branch of $M$ at T. If $S$ is one of the base points $P_{i}$, then the same assertion holds for the set of curves $\Gamma \in \mathfrak{N}$ which are tangent to one of the two branches of $J$ at $S$.

Proof. A line $\ell$ through $T$ corresponds to a linear pencil $\mathfrak{L}$ contained in $\mathfrak{N}$. The intersections of $\ell$ with $M$ correspond to the singular curves in the pencil. Their number $\delta$ is given by formula (8) in $\S 1 \mathrm{~B}$. Suppose $T$ is smooth on $M$. Then saying that $\ell$ is tangent to $M$ at $T$ means that the curve $\Gamma \in \mathfrak{N}$ which is associated with the point $T \in \mathbb{P}^{2}$ must be counted twice in the enumeration. When does this happen? Enriques and Chisini [2, L.3, Chapter 2, $\$ 20$, pp. 160-167] present a long discussion of the matter, which leads to the following conclusion: the double point $S$ of $\Gamma$ is a fixed point of the pencil, i.e. $\mathfrak{L}$ consists precisely of the curves $\Gamma \in \mathfrak{N}$ that pass through $S$. This proves the lemma when $T$ is smooth.

The case in which $T$ is singular on $M$ is more subtle. One must remember that $\delta$ is not so much the number of singular curves in the pencil, rather it is the number of double points belonging to curves in the pencil. Indeed a curve with two double points must be counted twice in the enumeration. Hence $M$ has three types of singularities: double points corresponding to the base points $P_{i}$, or to curves having two double points; cusps corresponding to the cuspidal curves of the net $\mathfrak{N}$. All these cases can be handled with the results of $[2$, loc. cit.]. Another approach would be to reduce to the preceding case by blowing up some points on $\mathbb{P}^{1} \times \mathbb{P}^{1}$, since the problem is essentially local in nature.

Let us now consider a curve $\Gamma_{2,3} \subset \mathbb{P}^{1} \times \mathbb{P}^{1}$ with two double points $S_{1}, S_{2} \in J$. The image $T=q \circ p^{-1}\left(S_{1}\right)=q \circ p^{-1}\left(S_{2}\right)$ is a double point of $M$ with two distinct branches. Indeed, it follows from Lemma 4.2 that $T$ can have two identical tangents only if all curves $\Gamma_{2,3}^{\prime}$ through $S_{1}$ also pass through $S_{2}$. But both $\Gamma_{2,3}$ and $\Gamma_{2,3}^{\prime}$ pass through the nine points $P_{1}, \ldots, P_{9}$; moreover, $S_{1}$ and $S_{2}$ are double on $\Gamma_{2.3}$; hence the intersection number of these two curves should be at least 13. This is impossible, since $\left(\Gamma_{2,3} \cdot \Gamma_{2,3}^{\prime}\right)=12$ and $\Gamma_{2,3}^{\prime}$ can be chosen irreducible (there are only finitely many reducible curves of type $(2,3)$ through nine generic points of $\left.\mathbb{P}^{1} \times \mathbb{P}^{1}\right)$.

Similarly, the images $q \circ p^{-1}\left(P_{i}\right)$ of the $P_{i}$ are ordinary double points of $M$, but $M$ also has a certain number of cusps, which correspond to the ramification points of $q$.

+ Set $v=1$ in the first two equations (30) and apply Cramer's rule. 
Lemma 4.3. The curve $M$ is irreducible, with degree 20 , geometric genus 9 , and class 8. Therefore it has 162 double points, among which are 114 nodes and 48 cusps. Nine of the nodes are the images of the base points $P_{i}$ of the net, and nine correspond to some reducible curves (of the form $\Gamma_{2,2}+\Gamma_{0.1}$ ). The remaining 96 nodes are in one-to-one correspondence with the irreducible curves of genus zero that belong to the net.

Proof. We have already shown that $M$ is irreducible. Since it is birationally equivalent to $J$, it has the same geometric genus, namely $p_{a}(J)-9=18-9=9$, for $J$ is of type $(4,7)$ and has nine ordinary double points (Lemma 4.1 ). The degree of $M$ is its intersection number with a generic line in the plane. Hence it is equal to the number $\delta$ of singular curves in a general linear pencil contained in $\mathfrak{N}$. In Lemma 1.3 we saw how to compute this number: $\left(\Gamma_{2,3}\right)^{2}=12, p_{a}\left(\Gamma_{2,3}\right)=2$ by Lemma 1.1; and formula (8) yields $\delta=20$.

Finally, we must evaluate the class of $M$. This is the number of tangents we can draw to $M$ from a generic point $T \in \mathbb{P}^{2}$. Let $\Gamma_{2,3} \in \mathfrak{N}$ be the curve which is associated with $T$. If $\ell$ is tangent to $M$ at $q \circ p^{-1}(S)$, it follows from Lemma 4.2 that $S \in \Gamma_{2,3}$. Hence $S \in \Gamma_{2,3} \cap J_{4,7}$. But a line drawn from $T$ through a double point of $M$ is not to be considered a tangent. The $9.2=18$ intersections of $\Gamma$ with $J$ at the base points $P_{i}$ must therefore be discounted and $S$ varies in a set of $26-18=8$ points. This shows that the class of $M$ is equal to 8 .

From what we know of $E$, it is clear that the curve $M$ has double points only, which are either nodes or ordinary cusps. Let $\delta$ be the number of nodes, and $\kappa$ that of cusps. By the Plücker formulae we have

$$
\begin{aligned}
& 9=p_{g}(M)=\frac{19.18}{2}-\delta-\kappa, \\
& 8=\operatorname{class}(M)=20.19-2 \delta-3 \kappa .
\end{aligned}
$$

Hence $\kappa=48$ and $\delta=114$. We have already seen that the nine base points $P_{1}, \ldots, P_{9}$ correspond to nodes of $M$. The other nodes correspond to curves $\Gamma_{2,3} \in \mathfrak{N}$ with two double points, but among them nine are reducible. They are composed of a quartic of genus 1 through eight of the $P_{i}$ and a straight line through the ninth point. These are the only reducible elements of the net.

REMARK. What do the 48 cusps correspond to? We shall not need this fact, and so we refer to [2, pp. 179-180] for details: among the curves of $\mathfrak{N}$, a finite number have a cusp somewhere on $\mathbb{P}^{1} \times \mathbb{P}^{1}$. These are the cusps of $M$. Their number happens to be equal to 24 times the geometric genus of a generic curve in the net (hence 48 in our case). Enriques and Chisini assert that this simple formula holds with great generality for all kinds of nets.

\section{Corollary 4.4. $\Delta_{2,3}=96$.}

This is formula (1). This method of proof can also be used to find some estimates for the other $\Delta_{\mu, v}$. Indeed let $N=(\mu-1)(v-1)$; this is both the arithmetic genus of $\Gamma_{\mu, v}$ and the dimension of the linear system $\mathfrak{M}$ we are interested in. The curves $\Gamma_{\mu, v}$ through $P_{1}, \ldots, P_{2 m-1}$ are in one-to-one correspondence with the points of $\mathbb{P}^{N}$. Those having at least one double point somewhere on $\mathbb{P}^{1} \times \mathbb{P}^{1}$ are mapped into the points of some hypersurface $M \subset \mathbb{P}^{N}$. This variety $M$ contains a flag of multiple subvarieties, which are associated with curves $\Gamma_{\mu, v}$ having more than one double point. In order to 
evaluate $\Delta_{\mu, v}$, we have to find the number of $N$-fold points on $M$ and to discount, among others, those associated with some reducible curves $\Gamma_{\mu, v}$. It is worth noting that $M$ is not an arbitrary hypersurface in $\mathbb{P}^{N}$ : one can easily determine its degree and some other invariants. Moreover, for $N>2$, it is always rational, since it is birationally equivalent to $\mathbb{P}^{1} \times \mathbb{P}^{1} \times \mathbb{P}^{N-3}$ (the curves with a double point at $S \in \mathbb{P}^{1} \times \mathbb{P}^{1}$ form a linear system of dimension $N-3$ ).

As an illustration, let us look very briefly at the number $\Delta_{2,4}$. In this case, the variety of moduli $M$ is a rational surface in $\mathbb{P}^{3}$. Its degree is equal to 28 , as follows again from formula (8). Its class is equal to 5 , because the Jacobian of a net containing a prescribed pencil $\{\lambda f+\mu g\}$ normally has singularities only at the base points of the net (Lemma 4.1.1), i.e. at the intersection points of the two curves $f$ and $g$ (but the eleven fixed points of the web $\mathfrak{M}$ have to be discounted). The rank (i.e. the class of a plane section) of $M$ is equal to 14 , as can be seen by an argument similar to the one used in Lemma 4.3: $\left(\Gamma_{2,4} \cdot J_{4,10}\right)-11.2=14$. Finally, the geometric genus of a plane section is equal to $p_{g}\left(J_{4,10}\right)=27-11=16$. From this we see that $M$ has a nodal curve of degree 263 and a cuspidal curve of degree 72 . By a classical formula in the theory of surfaces $[9, \# 627$; or 7 , Theorem 4 ; also 6 , formula $(\mathrm{V}, 83)]$ we find that $M$ has at most $\left[\frac{1}{3} \cdot 263 .(28-2)\right]=2279$ triple points; hence

$$
\Delta_{2,4} \leqslant 2279 .
$$

This is only a rough estimate. A very careful study of the surface $M$ is in order if we want to find the true value of $\Delta_{2,4}$. For instance, the nodal curve is not irreducible: it contains at least eleven straight lines, which correspond to divisors of the form $\Gamma_{2,3}+\Gamma_{0,1}$ on $\mathbb{P}^{1} \times \mathbb{P}^{1}$ (there are $\infty^{1}$ curves of type $(2,3)$ through $P_{1}, \ldots, P_{10}$; the line $\Gamma_{0,1}$ contains the remaining point $\left.P_{11}\right)$. Moreover, each of these lines contains twenty triple points of $M$ (by formula (8) again). Hence $\Delta_{2,4}$ should not exceed 2000 or so. But it is not our purpose to complete the determination of $\Delta_{2,4}$. We only wanted to point out that there is a very rich structure associated with the hypersurfaces of moduli. The following question appears as the natural outcome of this discussion.

Problem. Find a good upper bound (depending on the degree and some other invariants of $M$ ) for the number of (non-isolated) $N$-fold points of a hypersurface $M \subset \mathbb{P}^{N}$.

Added in proof. A recent paper of I. Vainsencher (Trans. Amer. Math. Soc., 267 (1981), 399-422) sheds some further light on the calculations of $\$ 4$. In particular, Vainsencher gives a proof, in a more general context, of Caporali's formula, mentioned in the Remark following Lemma 4.3. In fact, for a general net of curves $\Gamma$ lying on an arbitrary smooth surface $Y$, it follows from his formula (8.6.3) that the number of cusps is equal to $24\left(p_{a}(\Gamma)+p_{a}(Y)\right)$.

Furthermore, Vainsencher's formula (8.3) implies that $\Delta_{2,4}=640$. Indeed, the number of curves in the web $\mathfrak{M}$ having three double points is equal to 860 (apply (8.3) with $Y=\mathbb{P}^{1} \times \mathbb{P}^{1}$ and $\left.M \sim \Gamma_{2,4}\right)$. But one must subtract the 220 reducible curves, of the form $\Gamma_{2,3}+\Gamma_{0.1}$ with a double point on $\Gamma_{2,3}$. Of course, applying Theorem 1, we obtain $P^{12}=16+4 \Delta_{2,4}=2576$. 


\title{
References
}

1. W.-L. ChOW and B. L. VAN DER WAERDEN, 'Zur algebraischen Geometrie IX. Über zugeordnete Formen und algebraische Systeme von algebraischen Mannigfaltigkeiten', Math. Ann., 113 (1937), 692-704.

2. F. EnRIQUes and O. ChISINI, Lezioni sulla teoria geometrica delle equazioni e delle funzioni algebriche, Vol. II (N. Zanichelli, Bologna, 1918).

3. H. Hironaka, 'On the arithmetic genera and the effective genera of algebraic curves', Mem. Coll. Sci. Kyoto, A30 (1957), 177-195.

4. W. V. Hodge and D. Pedoe, Methods of algebraic geometry, Vol. 2 (Cambridge University Press, 1952).

5. S. L. Kleiman, 'The transversality of a general translate', Compositio Math., 28 (1974), 287-297.

6. S. L. Kleiman, 'The enumerative theory of singularities', Real and complex singularities, Oslo 1976 (Sijthoff and Noordhoff, Alphen aan den Rijn, 1977), pp. 297-396.

7. R. PIENE, 'Some formulas for a surface in $\mathrm{P}^{3}$ ', Algebraic geometry, Tromso 1977, Lecture Notes in Mathematics 687 (Springer, Berlin, 1978), pp. 196-235.

8. 1. R. ŠAfAREvič, Basic algebraic geometry (Springer, Berlin, 1974).

9. G. SAlmon, A treatise on the analytic geometry of three dimensions, 3rd edn (Hodges, Smith and Co., Dublin, 1874).

10. P. SAMUEL, 'Rational equivalence of arbitrary cycles', Amer. J. Math., 78 (1956), 383-400.

11. H. SCHUBERT, Kalkül der abzählenden Geometrie (Teubner, Leipzig, 1879).

12. J. G. Semple and L. Roth, Introduction to algebraic geometry (Clarendon Press, Oxford, 1949).

13. R. Sturm, 'Erzeugnisse, Elementarsysteme und Charakteristiken von cubischen Raumcurven', J. Reine Angew. Math., 79 (1875), 99-139; 80 (1875), 128-149.

14. J. A. Todd, 'On twisted cubic curves which satisfy twelve conditions', Proc. Roy. Soc. London Ser A, 131 (1931), 286-306.

15. B. L. VAN DER WAERDEN, Einführung in die algebraische Geometrie (Springer, Berlin, 1939).

16. A. WeIL, Foundations of algebraic geometry, 2nd edn (American Mathematical Society, Providence, R.I., 1962).

17. O. Zariski, Algebraic surfaces (Springer, Berlin, 1935, 1971).

18. O. Zariski, Collected Papers, Vol. II (Massachusetts Institute of Technology Press, Cambridge, Mass., 1973).

\author{
Université de Genève \\ Section de Mathématiques \\ 2-4, rue du Lièvre \\ CH-1211 Genève 24 \\ Switzerland
}

